REVIEW

\title{
Radionuclide carriers for targeting of cancer
}

\author{
Stavroula Sofou \\ Laboratory for Drug Delivery \\ Systems, Othmer-Jacobs Department \\ of Chemical and Biological \\ Engineering, Polytechnic University, \\ Brooklyn, NY, USA
}

\begin{abstract}
This review describes strategies for the delivery of therapeutic radionuclides to tumor sites. Therapeutic approaches are summarized in terms of tumor location in the body, and tumor morphology. These determine the radionuclides of choice for suggested targeting ligands, and the type of delivery carriers. This review is not exhaustive in examples of radionuclide carriers for targeted cancer therapy. Our purpose is two-fold: to give an integrated picture of the general strategies and molecular constructs currently explored for the delivery of therapeutic radionuclides, and to identify challenges that need to be addressed. Internal radiotherapies for targeting of cancer are at a very exciting and creative stage. It is expected that the current emphasis on multidisciplinary approaches for exploring such therapeutic directions should enable internal radiotherapy to reach its full potential.
\end{abstract}

Keywords: cancer radiotherapy, targeted radiotherapy, radiotherapy of micrometastases, radiotherapy of solid tumors, radiotherapy of tumor vasculature

\section{The drug}

In internal radionuclide therapy, as opposed to external beam therapy, the radiations of interest are primarily particle emissions (alpha-, beta-particles, and Auger electrons; Table 1; see Kassis and Adelstein 2005), that aim on the destruction of the nuclear DNA strands by radiation-induced ionizations, excitations, chemical transmutations and local charge effects (Nunez et al 1995). During recoil, the ionizing radiation produces tracks along which energy is transferred to the surroundings. Linear energy transfer (LET) describes the transferred energy per distance traveled by the emitted particles. Alpha particles have high LET and produce densely ionizing tracks with highly lethal effects. Beta particle emitters, due to lower LET, produce sparsely ionizing tracks with lower killing efficacy. Beta particles have the longest range in tissue followed by alpha particles and Auger electrons. The range of Auger cascade electrons are just of the order of a few nanometers, essentially creating a very localized irradiated area (sphere) around the decay site of the parent radionuclide (Zaidi and Sgouros 2003). The range of these emitted particles in tissue is a great determinant of the size of tumors to be potentially treated, and affects other considerations regarding the type of targeting ligand and delivery carrier for the parent radionuclide.

Specifically, alpha particles are positively charged monoenergetic helium nuclei with the highest energy among particle emissions ranging from 5 to $9 \mathrm{MeV}$. Alpha particles are characterized by LET values of the order of $80 \mathrm{keV} / \mu \mathrm{m}$ along the straight line tracks they travel with rate of energy deposition that increases approximately to $300 \mathrm{keV} / \mu \mathrm{m}$ at the end of the track. Their range in tissue varies between 5 and 10 cell diameters (40 to $100 \mu \mathrm{m}$ ) depending on their energy (Kassis and Adelstein 2005). This is consistent with the dimensions of micrometastatic disease, allowing for localized irradiation of target cells with minimal toxicity on the surrounding normal cells. Alphas are particularly effective in cell killing (McDevitt et al 2001): cell survival studies have shown that alpha particle induced killing is independent of oxygenation state or cell cycle phase during irradiation, and that as few as 1-3 tracks across the 
Table I Physical characteristics of therapeutic radionuclides

\begin{tabular}{llll}
\hline & Particles & Energy $\mathbf{E}_{\min }-\mathbf{E}_{\max }$ & Range \\
\hline$\beta$ & Electrons & $\begin{array}{l}\text { Medium to high* } \\
(0.05-2.3 \mathrm{MeV})\end{array}$ & $0.2-12 \mathrm{~mm}$ \\
$\alpha$ & Helium nuclei & High** $5-9 \mathrm{MeV}$ & $\begin{array}{l}40-100 \mu \mathrm{m} \\
\text { EC/IC }\end{array}$ \\
& Auger electrons & $\begin{array}{l}\text { Very low** } \\
(\mathrm{eV}-\mathrm{keV})\end{array}$ & Several $\mathrm{nm}$ \\
& & & \\
\hline
\end{tabular}

Abbreviations: EC, electron capture; IC, internal conversion.

Notes: *average values; **monoenergetic.

nucleus may result in cell death (Humm 1987; Macklis et al 1988; Humm and Chin 1993; Couturier et al 2005) causing single- and double-stranded DNA breaks. Studies on survival of normal human epithelial cells suggest that alpha particle traversals in the cytoplasm or tangential to the cell nucleus do not affect cell survival (Søyland and Hassfjell 2000). These studies propose the significance of the alpha particle traversed path length in the cell nuclei rather than the number of alpha particle traversals of cell nuclei. Consequently, internalization of alpha particle emitters by cancer cells increases the chance to kill: alpha particles originating on the cell surface have only an estimated $30 \%$ probability of traversing the cell nucleus (Nikula et al 1999).

Beta particles are negatively charged electrons and their range in tissue is of the order of a centimeter that is comparable with larger size tumors. Beta particles have energies ranging from 0.05 to $2.3 \mathrm{MeV}$ with LET of the order of $0.2 \mathrm{keV} / \mu \mathrm{m}$ (Table 1). Consequently, disregarding other parameters such as tumor penetration profiles of radionuclide carriers, higher radionuclide concentrations of beta emitters compared to alpha emitters are required for comparable cell kill (Zaidi and Sgouros 2003). However, the long range of beta particles results in a "cross fire" effect. This effect is due to the long beta particles' path that crosses multiple individual cells. The cross fire effect decreases the need to target each cancer cell with a radionuclide emitter. Cell survival studies show that cells are more sensitive to beta radiation when they are arrested in the G2 phase, and are in a good oxygenated state. The presence of oxygen upon beta particle irradiation generates free radical species that are particularly harmful to the nuclear DNA. Both the rate of beta particle disintegration (half-life) and number of beta emitters (radioactivity) at the target site influence the killing efficacy by competing against the enzymatic repair of the irradiated DNA strands (Zaidi and Sgouros 2003).

Auger electrons are low energy atomic orbital electrons emitted after electron capture (Kassis et al 1987; Kassis 2004). During nuclear recoil they produce an array of reactive radicals similar to those formed by alpha particle tracks.
Because of the nanometer range tracks, the precise subcellular localization of Auger emitters can dramatically affect their killing efficacy. Nuclear localization results in significant enhancement of killing compared to localization at the cell surface (Faraggi et al 1994), making the subcellular accumulation sites of Auger emitters of paramount importance on their killing efficacy (Boswell and Brechbiel 2005).

Tumor response to particle radiation depends on several parameters including the absorbed dose, the dose rate, the tumor penetration profile of radionuclides, the intracellular localization profiles of radionuclides of shorter range, and the tumor radiosensitivity.

Calculation of the absorbed dose (D) is necessary to quantitatively correlate tumor response to a particular radiotherapeutic modality and to project on the potential effect of other radiotherapeutic modalities or administration strategies. In simple terms, the absorbed dose from a target site is defined as the energy (E) absorbed by a particular mass of tissue, normalized by the tissue mass $(\mathrm{M}): \mathrm{D}=\mathrm{E} / \mathrm{M}$ (Sgouros 2005). The absorbed energy is defined as a function of three parameters: the number of disintegrations within the particular volume of interest ( $\delta$ ), the energy emitted per disintegration $(\varepsilon)$, and the fraction of emitted energy that is absorbed by the particular volume of interest (the target mass) (f): $E=\delta \times \varepsilon \times f$. For the relatively long range beta emitters, the dose evaluation at a target site includes not only the energy emitted by radionuclides localized within the target volume, but also the energy emitted by radionuclides accumulated in neighboring organs or areas whose emissions cross along their path the target volume of interest (Kolbert et al 2003). In other words, the calculated total absorbed dose is the sum of the dose contributions from all regions containing radionuclides that act as secondary sources. The adsorbed dose due to photon emissions is usually calculated separately and added to the dose due to alpha or beta particles. For alpha particle emitters such cross organ absorbed doses may be of no significance due to their short recoil distances. At the micron-scale and at distances comparable to a few cells, microdosimetric evaluations are used to evaluate dose or 'hits' acquired by cancer cells within micrometastatic clusters (Palm et al 2002).

Evaluation of the rate of clearance and rate of uptake at the target site rather than only the total accumulation of a radiopharmaceutical at key body sites (bone marrow, liver, spleen etc) can provide direct information to mathematical modeling for estimation of useful quantities in dosimetry such as antigen density (for radioimmunotherapy), tumor burden and antigen availability (Kolbert et al 2001). 
The apparent lethal efficacy of the calculated self-dose and cross-dose may also be influenced by the bystander effect. This effect describes the enhanced killing of cells by radiation and even of cells that have not been accessed by the radionuclides. It is believed to be a biological effect that has been shown to be proportional to the dose that is delivered to the neighboring targeted cells, and has been primarily studied and verified in vitro for alpha particle-, beta particle- and Auger electron-emitters. Depending on the radiation's LET, this effect has been suggested to be driven by gap junction intracellular communications or by secretion from irradiated cells of cytokines and free radicals. These secreted compounds are taken up by non-irradiated cells that, in turn, respond with reduced survival (Snyder 2004). Recently, evidence for the bystander effect of Auger electron-emitters in vivo was also reported (Xu et al 2002). It is possible that for certain cases omission to account for the bystander effect in the dose determination could interfere with the correct evaluation of the dose effect. This is because of the way the dose is traditionally determined leading to underestimation of the radiobiological effect (Hei et al 2004; Kassis 2004). Currently, the molecular and physical mechanism(s) that describe the bystander effect are not fully understood. Elucidation of these mechanisms should provide the tools to mathematically incorporate their effect on dose estimation (Sgouros et al 2007).

Depending on the radiosensitivity of the target site, radiosensitizers for beta-particle therapy are also employed to improve killing efficacy. Major design properties of an ideal radiosensitizer and its delivery vehicle include: the appropriate timing of the radiosensitizer's administration and its presence at the tumor microenvironment relative to the therapeutic radiation, the radiosensitizer's selective tumor accumulation and its adequate tumor localization and retention. Minimal toxicity and minimal enhancement of radiation toxicity at normal organ sites are major considerations (for a review see Kvols 2005). Compounds currently in use include molecules that either target DNA (thymidilate synthase inhibitors or platinum analogues), or aim at non-DNA targets. p53-mediated radiosensitization was also reported using folate-targeting liposomes for wtp53 transfection (Xu et al 2001). Examples of the non-DNA targeting category include compounds for targeting the epidermal growth factor receptor (EGFR), inhibitors of the Ras function, prostaglandin inhibitors or compounds that target the tumor neovasculature inhibiting angiogenesis and tumor nutrient- and oxygensupply. Contrary to beta particles, complete understanding of potential radiosensitization mechanisms towards alpha particles requires further studies (Supiot et al 2005, 2007).
When radionuclide delivery carriers are designed to specifically and directly target cancer cells (or endothelial cells of the tumor neovasculature), several criteria need to be evaluated regarding the molecular targets, including their expression by cancer cells relative to non-targeted cells and their relationship to the cell cycle, their surface density and their homogeneity on the cell surface. Several types of molecular targets used in targeted internal radiotherapy will be presented throughout the present review. Characteristic types include regulatory peptide receptors like the somatostatin receptors for peptide receptor radiation therapy (PRRT) (Reubi et al 2005), EGFR (Hynes and Lane 2005; Normanno et al 2006), antiangiogenic molecular targets (vascular endothelial growth factor receptors [VEGFR]) (Alessi et al 2004), integrins in antivascular therapies, and other novel targets under examination (Britz-Cunningham and Adelstein 2003). Correspondingly, the choice of the targeting ligand ideally is based on its binding specificity to the chosen molecular targets and stability in vivo, the target affinity and stability of the ligand-labeled carriers, and their resistance to degradation due to radiation. Stably radiolabeled constructs are crucial. For extensive discussions on radiolabeling strategies the readers are referred to reviews specific to the subject (Adam and Wilbur 2005; Brechbiel 2007).

\section{The carriers}

The types of radionuclide carriers reviewed here are categorized based on the disease topology and/or tumor morphology and not based on the type of construct (for example liposome- or polymer- or antibody-based). The rationale for this approach is to better describe and group the particular characteristics of the targeted disease that accordingly influence the design and engineering of delivery carriers.

Metastatic cancer can exist on the same host at different stages simultaneously: ranging from single cancer cells or micrometastatic tumors to solid tumors. Micrometastases refer to small avascular cancer cell clusters. By solid tumors larger vascularized multicellular clusters are implied. To result in high tumor absorbed doses with relative low normal organ accumulation, a variety of designs for targeting carriers combined with different radionuclides are proposed. This variety emerges from the numerous variables describing each case. Such variables include the type (cancer biology) of cancer cells that constitute the tumors that would determine for example the relevant biomolecular markers, the tumor's anatomic location in the body (topology), the shape and size of individual tumors (morphology), the presence or not of direct access routes to tumors such as vasculature or direct 
administration in the space where they may be confined (blood or the peritoneal cavity), and others. Independently of the type (molecular type, and size) of the delivery carrier, stability of radionuclide conjugates is of paramount importance. Released radionuclides are free to distribute throughout the body and potentially accumulate at normal organs increasing toxicity.

In vascularized tumors, the so called "active" targeting of drug carriers is directed by specialized targeting ligands that aim at the molecular targets located on the surface of either the endothelial cells of tumor's neovasculature or of the cancer cells that constitute the tumors. On the contrary, "passive" delivery of drug carriers to vascularized tumors denotes the extravasation from the blood stream into the tumor interstitium, and the preferential retention by tumors of nanometer-sized drug carriers compared with smaller compounds. The Enhanced Permeability and Retention (EPR) effect that describes this mechanism is due to a combination of the tumor leaky neovasculature (Hobbs et al 1998; Jain et al 2002), the malfunctioning lymphatics, and the high interstitial pressures, and has been verified experimentally by several groups (Hobbs et al 1998; Noguchi et al 1998). Nanometer sized carriers or macromolecules exhibiting enhanced retention by vascularized tumors are ideal for selective tumor accumulation (Noguchi et al 1998; Maeda et al 2000). In xenografts of human and murine tumors, the vascular reported pore cut off sizes can range from $200 \mathrm{~nm}$ to $1.2 \mu \mathrm{m}$ (Hobbs et al 1998). And because passive tumor accumulation is a random event, for tumor accretion to become clinically compelling, blood circulation of carriers needs to be extensively sustained so as to increase the probability that the carrier will encounter the tumor. Extensive increase of the drug carriers' circulation times with impressive improvement of their biodistributions has been achieved by grafting polyethylene glycol polymers (PEGylation) on proteins, macromolecules and supramolecular drug carriers. It is believed that PEG acts as a steric barrier and interferes in the interactions of the carriers with serum proteins, cells of the immune- and reticuloendothelial-systems, and with other carriers (Lasic and Martin 1995; Allen et al 2002; Auguste et al 2003; Gabizon et al 2003; Sofou 2007). In internal radionuclide therapy the physical half-life of the delivered therapeutic radionuclides can be used as a free parameter to optimize the tumor delivered dose with respect to normal organ irradiation.

In micrometastatic disease, direct routes of administration at the cancer cells' microenvironment are required to present the carrier and targeting moiety in the close vicinity of the tumor. Adsorption and internalization of radionuclide carriers by cancer cells that constitute the micrometastatic tumors, as well as their diffusion through the micrometastasis' interstitial space and their penetration towards the micrometastatic core are characteristics that depend on several interfacial events. These include the binding strength of targeting ligands to cell surface receptors, potential nonspecific attractive interactions between the cell surface and the radionuclide carrier surface, and the size of the radionuclide carriers (Allen 2002; Emfietzoglou et al 2005). Alpha- or Auger- emitters, due to their short range in tissues, would be more appropriate for effective killing of circulating single cancer cells with minimal irradiation of the blood vessels.

In all of the above cases, however, a major limitation in the therapy of cancer using internal radiotherapy still remains the dose-limiting toxicity. This is due to the relatively significant accumulation of radionuclide carriers in vital organs compared to target sites. It is the toxicity that prohibits administration of higher doses that could reach lethal absorbed levels at the tumor sites. Approaches to address this challenge include: direct single-step targeting using delivery carriers that result in improved biodistributions, strategies to stop angiogenesis without affecting normal tissues, direct selective targeting and destruction of neovasculature, "normalization" of the tumor neovasculature to enhance penetration of the drug into the tumor heterogeneous interstitial space, tumor accumulation of inactive prodrugs and localized "meta-activation" after localization at the tumor, direct targeting of easily accessible cancer cells, multi-step targeting to dissociate radiotoxicity from carrierinduced toxicity, and others. Examples of these approaches are discussed below.

\section{Single-step tumor accumulation}

Here we report on radionuclide carriers without tumorspecific ligands on their surface. These carriers are designed to either passively accumulate within the tumor interstitial space following extravasation from circulation in the blood stream, or to become physically entrapped into the fenestrations of the diseased tissue such as the liver. Magnetic radionuclide carriers that are "magnetically targeted" are also included in this section.

For vascularized tumors, passive accumulation within the tumor interstitial space is primarily influenced by the size and circulation time of the nanometer sized carriers. Liposomes with encapsulated radionuclides have been extensively studied (Hwang et al 1982; Henriksen et al 2004b; Sofou 
et al 2004b). Liposomes are closed shell structures defined by one or more bilayer membranes that enclose an aqueous interior. The membranes consist of amphiphilic phospholipids (double-tailed, single-headed molecules) that self-assemble in water. Phospholipid bilayer membranes have a thickness of about $4 \mathrm{~nm}$, and liposomes may entrap thousands of water soluble molecules in their internal aqueous compartment (Lasic 1993). The advantage of liposome-based carriers, apart from altering the biodistributions of the radiopharmaceutical, is their high drug-to-carrier ratios. High encapsulation efficiencies of radionuclides by liposomes are achievable. Active loading protocols on preformed liposomes have been reported in the literature (Hwang et al 1982; Henriksen et al 2004a). Due to the current trend in liposome research for biomedical applications, towards using higher Tg lipid membranes, understanding the mechanisms for high radionuclide entrapment efficiencies is of great importance. Liver and spleen are common accumulation sites for liposomes. Therefore, for radionuclide delivery to solid tumors residing in these organs, passive accumulation of radiolabeled liposomes could be a logical choice. Alternatively, pretreatment with non radioactive liposomes may saturate the hepatic macrophages resulting in lower liver uptake of the subsequently administered radiolabeled liposomes (Jonasdottir et al 2006).

Dextrans loaded with radionuclides have also been extensively studied for passive accumulation at the sites of vascularized tumors. Dextrans are branched polysaccharides composed of glucose molecules joined into chains of varying lengths. They have been in clinical use for several decades mainly to decrease vascular thrombosis. Similarly to liposomes, dextran's size that is of a few nm's determines their localization in the body that is commonly at the liver and spleen. Extensive radiolabeling of dextrans and stable retention of radionuclides is critical for the effectively delivered tumor dose and the potential toxicity sites in vivo. Stable radiolabeling of dextrans with the beta particle emitter Rhenium-188 $\left(\mathrm{t}_{1 / 2}=16.9 \mathrm{~h}, \mathrm{E}_{\max }=2.11 \mathrm{MeV}\right.$, maximum range of $11 \mathrm{~mm}$ in soft tissue) was studied by Du and colleagues (2000). It is suggested by the authors that ${ }^{188} \mathrm{Re}$ is particularly useful because it also emits gamma photons of $155 \mathrm{keV}$ that enable monitoring of uptake and dosimetry calculations. However, stability of the rhenium radiocomplexes has been reported to be an issue in vitro owed to rhenium's reoxidation and transchelation. Du and colleagues (2000) modified dextran's surface by cysteine and used the free thiols for radiolabeling with ${ }^{188} \mathrm{Re}$-gluconate as transchelator. This approach exhibited $60 \%-70 \%$ labeling efficiency. In physiological conditions $-5 \%$ human serum albumin in $0.9 \%$ saline solution at $37{ }^{\circ} \mathrm{C}$ - the radiolabeled dextrans released less than $15 \%$ of conjugated radioactivity for 24 hours in the presence of ascorbic acid under a nitrogen atmosphere.

In a different approach for passive accumulation of radionuclide carriers at vascularized hepatic tumors, hepatic arterial injection of micrometer sized particles has been used. In this approach, particles loaded with radionuclides are administered for hepatic embolization against primary or metastatic liver tumors. Locoregional administration into the hepatic artery results in the majority of injected activity to remain within the liver that provides almost exclusively the blood supply to hepatic metastases. Fortunately, normal liver tissue receives only $30 \%$ of its blood supply from the hepatic artery. Materials used for such microspheres include ceramics, polymers, resins or glass (Mantravadi et al 1982; Lin et al 1984). Increase of the tumor-to-liver ratio of absorbed activities beyond unity is the major requirement for minimizing toxicity at the liver parenchymal cells. The treatment efficacy seems to depend strongly on the vascularity of the hepatic malignancies (Mantravadi et al 1982), and it was reported that micrometer sized spheres may be too large to be dispersed into the hepatic tumor interstitium. Use of smaller size microspheres has been shown to be more effective at least in rats (Haefeli et al 1999). Reported challenges of this approach include heterogeneous microsphere distribution with high accumulation near the periphery of tumor vasculature and less localization at the tumor center, as well as clustering of deposited microspheres (Campbell et al 2000). In addition, microspheres can potentially be released from the liver. In such cases, they commonly accumulate in the lungs. Microspheres bearing beta particle emitting radionuclides, mainly ${ }^{90} \mathrm{Y}\left(\mathrm{t}_{1 / 2}=2.67\right.$ days, $\left.\mathrm{E}_{\max }=2.28 \mathrm{MeV}\right),{ }^{186} \mathrm{Re}$ $\left(\mathrm{t}_{1 / 2}=3.78\right.$ days, $\mathrm{E}_{\max }=1.077 \mathrm{keV}$, maximum range of $6 \mathrm{~mm}$ in soft tissue) and ${ }^{188} \mathrm{Re}$ have been reported. Rhenium has the advantages of shorter required neutron activation times compared to ${ }^{90} \mathrm{Y}$, and the ability to allow for imaging. This is due to the gamma emissions which constitute $9.5 \%$ for ${ }^{186} \mathrm{Re}$ and $15.0 \%$ for ${ }^{188} \mathrm{Re}$ emissions with energies 137 and $155 \mathrm{keV}$, respectively. Clinical trials with ${ }^{90} \mathrm{Y}$-microspheres show encouraging results both for primary and metastatic liver tumors (Houle et al 1989; Salem et al 2002; Murthy et al 2005). Microspheres contain rhenium metal or ${ }^{89} \mathrm{Y}$ without chelation. Generally, small amounts of radioactivity are reported to leak from microspheres in vitro. Rhenium compounds released from glass microspheres have been reported to accumulate in the thyroid. For ${ }^{90} \mathrm{Y}$, bone marrow accumulation has been reported that could lead to myelosuppression (Haefeli et al 1999). 
Magnetic targeting has also been explored as an alternative strategy to enhance tumor accumulation with the objective to minimize systemic toxicity. Magnetic microspheres containing therapeutic radionuclides were used in this approach, and enhancement of localization of the delivery carriers at the tumors is achieved by using external magnetic fields. Hafaeli and colleagues (1995) synthesized micron-size poly(lactic acid) spheres with incorporated magnetite and the beta particle emitter ${ }^{90} \mathrm{Y}$, and demonstrated enhanced killing efficacy of neuroblastoma cells when a magnet was used to direct the microspheres closer to cells in vitro. In vivo studies showed high tumor accumulation by magnetic poly(lactic acid) radiomicropheres when directed towards a subcutaneous tumor by an external fixed magnet after intraperitoneal administration. Stable radiolabeling of magnetic nanoparticles with ${ }^{188} \mathrm{Re}$ is reported (Haefeli et al 2001). In a different approach, Chunfu and colleagues (2004) developed $200 \mathrm{~nm}$ particles composed of magnetite nanoparticles covered with human serum albumin and labeled with ${ }^{188} \mathrm{Re}$ via its sulfur surface atoms. These nanoparticles showed stable radionuclide retention in albumin solution for up to 72 hours.

After extravasation of radionuclide carriers into solid tumors, diffusion is the main transport mechanism that governs their penetration and dissemination throughout the tumor's interstitial space. Tumor interstitial diffusion is hindered by the extracellular matrix (ECM) comprising primarily collagen and glycosaminoglycans (such as hyaluronan). The effect of these biomacromolecules on the transport characteristics of antibodies and liposomes has been extensively studied (Netti et al 2000; Znati et al 2003). $\mathrm{X}$-ray radiation may further hinter the interstitial diffusion. In particular, interstitial diffusion may be further decreased due to the tumor levels of collagen type I within the tumor interstitium that seem to increase due to irradiation. It has been suggested that collagen binds and stabilizes the glycosaminoglycan component of the ECM resulting in increased resistance to the transport of macromolecules (Netti et al 2000). In vivo, decreased diffusion that was attributed to the increased levels of collagen type I was measured after external irradiation in subcutaneous implants of human colon (Znati et al 2003).

However, extravasation of the circulating drug carrier into the tumor interstitium depends on the endothelial wall permeability, and it precedes the carrier's diffusion and penetration into the tumor's core space. Kalofonos and colleagues (Kalofonos et al 1990) showed that vascular permeability of the tumor endothelium in human colon tumor xenografts significantly increases soon after exposure to X-ray irradiation.
However, this lasts only for short time periods. Therefore, the same type of radiation can differently affect the accumulation of radionuclide carriers to vascularized tumors by transiently increasing the endothelial wall permeability and by enhancing ECM's structural integrity. Timing of irradiation with respect to drug carrier administration was shown to play an important role in the therapeutic effects of liposomally encapsulated doxorubicin on human osteosarcoma xenografts when combined with external radiation. In this study, improved intratumoral distribution and tumor uptake was shown that can be achieved with appropriate timing (Davies et al 2004).

Other approaches to improve penetration of macromolecular delivery carriers in vascularized solid tumors include administration of enzymes that can degrade the ECM. Towards this goal, collagenase (Eikenes et al 2004) and hyaluronidase (Eikenes et al 2005) were evaluated in vivo on human osteosarcoma xenografts. In particular, before administration of an osteosarcoma specific monoclonal antibody, collagenase was administered intravenously. Although the particular enzyme is not clinically relevant because it may favor metastasis, a twofold increase in the tumor uptake of the osteosarcoma specific antibody was observed. In addition, the antibody exhibited improved penetration in the tumor. Using larger drug carriers, Eikenes and colleagues (Eikenes et al 2005) showed significant enhancement in tumor accumulation of intravenously administered liposomal drug carriers. This was achieved by administering intratumorally, preceding the administration of liposomes, the ECM enzyme hyaluronidase that degrades the extracellular tumor matrix. The adjuvant use of hyaluronidase has already been used in phase I and II trials to improve prevention of tumor regrowth when administered with chemotherapy. Surprisingly, intravenous administration of the enzyme was not found to be toxic to normal tissues (Bruera et al 1999). Such strategies of EMC pretreatment could, in principle, be applied to other types of macromolecular and nanometer sized delivery carriers in internal radiotherapy for improvement of the carrier's distribution within solid tumors.

In solid tumors, the irregular tumor vasculature may lead to heterogeneous distributions of the radionuclide carriers resulting in variable microdosimetric distributions. The significance of this effect to the delivered dose depends on the tumor size relative to the range of the emitted particles in tissue. Nonspecific interactions between the delivery carriers and cancer cells in tumor spheroids that may contribute to these irregularities were studied for liposomes and the role of electrostatics and steric repulsion (PEGylation) was 
shown to be significant (Emfietzoglou et al 2005). When targeting ligands such as antibodies are used, high affinities for the cell receptors, fast antigen internalization and slow diffusion were shown to result in high accumulation only at the perivascular regions (Adams et al 2001). A recent theoretical study on evaluating the criteria for efficient antibody penetration into solid tumors and micrometastases suggests that because of the "binding site barrier" antibodies could possibly be more suitable for targeting micrometastases (Thurber et al 2007).

\section{Targeting of tumor vasculature}

Receptors overexpressed on the endothelial cells comprising the tumor neovasculature are used as targets for therapy and diagnosis. Development of vasculature is needed for solid tumors to grow beyond $1-2 \mathrm{~mm}^{3}$ in size (Folkman 1990), and endothelial vascular cells in malignant state will divide as fast as every 7-20 days as opposed to every 20 years when in a healthy state (Hobson and Denekamp 1984; Scott and Harris 1994). In some cases of solid tumors, direct targeting of the neovasculature itself with radionuclides could be more attractive and efficient than targeting molecular markers on the surface of cancer cells that reside within tumors. In the latter case, the radionuclide conjugates will have to overcome additional barriers related to extravasation and diffusion/ penetration within the tumor interstitium.

The therapeutic strategies that involve targeting of the tumor neovasculature are categorized as antiangiogenic (or angiostatic) and as antivascular (or angiolytic). Antiangiogenic refers to the prevention of new blood vessel formation and includes targeting of growth factor receptors on the vasculature endothelial cells. Antivascular refers to damaging and killing of tumor cells by cutting the blood flow via the neovasculature, so as to deprive the tumor of growth factors (Folkman 1971), using as targets other characteristic markers such as integrins.

Healthy vasculature is composed of endothelial cells that create the walls of the vascular lumen. Endothelial cells are surrounded by pericytes and a basement membrane. Pericytes interfere with endothelial cell proliferation, microvessel outgrowth, and stabilization of the capillary walls (Sims 1986). Angiogenesis is the formation of new vasculature. Tumor vessels are abnormal in many respects. They may lack perivascular cells that protect vessels from changes in oxygen and hormonal levels, and the vessel wall itself might contain not only endothelial cells but also cancer cells resembling more of a mosaic. Choosing targeted marker molecules should be influenced by this architecture (Carmeliet and Jain 2000).
The rationale for targeting the tumor vasculature is based on the observation that without adequate vasculature tumor cells are shown to become necrotic or apoptotic (Burrows and Thorpe 1993; Brooks et al 1994). However, studies on targeting tumor vasculature have shown that by depriving tumors of oxygen, tumor cells may transform into hypoxic cells and consequently become more resistant to chemotherapy and radiation (Bottaro and Liotta 2003). Hypoxic cells can activate the production of proteins (hypoxia-inducible factor [HIF]) that are involved in VEGF/VEGFR-2 signaling, and increase the formation of protease enzymes that degrade the basement membrane and the extracellular matrix, thus promoting in this way cell mobility and invasion, and resulting in metastasis of cancer cells, and migration of the endothelial cells into the tumor interstitial space where they proliferate and create new vasculature.

Characteristic molecular targets that have been studied and extensively utilized include: the VEGFR, integrins, matrix metalloproteases, and other endogenous antiangiogenic factors. Matrix metalloproteases are neutral endopeptidases that can degrade the ECM. They interact with integrins and the endothelial growth factors. Matrix metalloproteinase inhibitors aim to inhibit the action of these metalloproteases. The cell surface proteoglycan NG2 marker of angiogenic pericytes (Burg et al 1999) or the oncofetal fibronectin that resides in the immediate ECM of blood vessels supplying malignant sites have also been suggested as alternative and very interesting targets (Ruoslathi 2002).

VEGF in particular, and its receptors, are important components in the process of angiogenesis. There are six growth factors in the VEGF family (the most well-characterized is VEGF-A) and three receptors which are transmembrane tyrosine kinases predominantly found in endothelial cells. VEGF is present in normal tissues and in tumors. As mentioned above, activation of the VEGFR-2 is connected to enhanced vascular permeability and proliferation of endothelial cells (Rosen 2005), and antibodies against VEGFR-2 have been explored as inhibitors of angiogenesis ( $\mathrm{Li}$ et al 2005). A systematic review of several studies on the combined administration of therapeutics to block proangiogenic factors (anti-VEGF) or administration of antiangiogenic factors with external radiation has been published by Nieder and colleagues (2006). The interaction mechanisms of tumor endothelial cells with antiangiogenic factors and ionizing radiation is particularly complex. Studies of such systems with internally targeted radionuclides whose action is not characterized by radioresistance, still needs to be explored (Wachsberger et al 2003). 
In antivascular strategies, targeting of integrins has attracted particular interest. Alpha v beta 3 integrin is expressed in newly formed endothelial cells and on endothelial cells of the neovasculature (Ruoslathi 2002). Its function is related to cell adhesion, and certain members of the integrin family are involved in cancer metastasis and angiogenesis. Briefly, integrins are bidirectional heterodimeric receptors consisting of one alpha- and one beta-subunit, and are activated by the tripeptide sequence Arg-Gly-Asp (RGD) (Ruoslathi and Pierschbacher 1986). Upon binding of an RGD-type peptide to a resting integrin, a series of signals is transduced that causes dissociation of alpha, beta- heterodimers and formation of clustered homooligomers (alpha-, and beta-oligomers). This surface nanopatterning creates adhesion focal points on the cell surface that is shown to lead to strong binding with multivalent ligands (multimeric RGD ligands) (Wester and Kessler 2005). When a competitive antibody or molecule bearing the RGD motif disrupts the integrin ligand interaction, blocking of formation of new blood vessels takes place, which is the basis for some antivascular therapeutics.

Janssen and colleagues (2002) studied an ${ }^{90} \mathrm{Y}$ labeled RGD-peptide to target the alpha $\mathrm{v}$ beta 3 integrin on the neovasculature of OVCAR-3 ovarian carcinoma xenografts. The study showed that the radiolabeled peptide delays tumor growth, but investigation of possible optimization of tumortargeting and tumor therapy using dose fractionation did not improve the therapeutic efficacy of the radiolabeled peptide (Janssen et al 2004).

In a different approach, because it is the larger tumors that develop neovasculature networks, combination of active targeting and passive targeting was studied by Line and colleagues (Line et al 2005). For active targeting multivalent RDG sequences were used that were attached on relatively large delivery carriers to also exploit passive targeting demonstrated by the EPR effect. In particular, a $30 \mathrm{kDa}{ }^{99 \mathrm{~m}} \mathrm{Tc}$-labeled HPMA copolymer was developed that was conjugated to RGD-containing peptides (Koivunen et al 1995), and was evaluated in two xenograft models of prostate cancer. The studies verified the additive effect of the two targeting mechanisms in tumor uptake, namely active and passive, but passive accumulation contributed to a lesser extent (Line et al 2005). Mitra and colleagues (Mitra et al 2006) also studied the antitumor effectiveness of a HPMA copolymer conjugated to multivalent RDG sequences and loaded with ${ }^{90}$ Y. Significant decrease in tumor volume was observed with increased apoptosis in treated tumors, and with no acute signs of toxicity to normal organs in a SCID mouse xenograft model of human prostate carcinoma. A combination of short range and long range particle emitters such as alpha and beta particles would be an interesting approach for such a construct. This suggestion is based on the assumption that the emitted alpha particles may have stronger killing impact on the endothelial cells, while the emitted beta particles are ideal for irradiating the cancer cells comprising the solid tumors.

The potential antivascular effect of alpha particle emitters has been proposed (Thorpe and Burrows 1995), and studied in vitro and in animals (Kennel and Mirzadeh 1998). Only recently, efficacious results were demonstrated in humans (Allen et al 2007). In particular, in a phase I trial of systemic melanoma the 9.2.27 monoclocal antibody was radiolabeled with Bismuth-213 $\left(\mathrm{t}_{1 / 2}=45.59 \mathrm{~min}\right)$ via cDTPA and was used to target the core glycoprotein of the melanoma associated chondroitin sulfate proteoglycan (MCSP). The studies showed partial or complete tumor regression in patients that was unexpected. This is because the alpha particle therapy was not intended for solid tumors but rather for circulating micrometastases. However, in the particular cancer, the targeted cancer cells are known to express the same MCSP antigen as the pericytes in solid tumors. Therefore, it was suggested that after extravasation of the radioimmunoconstructs into the solid tumor and binding to pericytes, the alpha particle emitters were localized not far from the vascular endothelial cells which they irradiated and killed. The authors concluded that the induced endothelial cell death resulted in closure of the capillaries and starvation of the solid tumors. Further investigation of this approach could result in alternative uses of alpha particle emitters.

Alternatively, an interesting approach to transiently produce vascular "normalization" followed by administration of chemotherapy or radiation has been proposed by Jain (2005). The rationale is based on the hypothesis that although antiangiogenic monotherapy causes destruction of the neovasculature, and higher doses should be more effective, high antiangiogenic doses may harm the vasculature of normal tissues as well, including the cardiovascular, endocrine, and nervous systems (Carmeliet and Jain 2000). In addition, because the blood supply network within the tumor is heterogeneous and interferes with our ability to deliver drugs into the tumors, administration of moderate antiangiogenic doses will not completely destroy the neovasculature. Also, in cases of combination therapies, it may leave the intratumoral drug delivery impaired. It is therefore suggested that "normalization" of the tumor vasculature should occur first by administering growth factors to create a homogeneous 
spatial vascular network within the tumor. In this way, tumor neovasculature will become less tortuous. Subsequently, and during the functional window where normalization occurs, administration of anticancer therapeutics should take place. This strategy could result in higher and more homogeneous uptake of therapeutics, which should be able to penetrate and disseminate deeper into the tumor interstitium and cells. Better drug distribution throughout the tumors combined with higher tumor oxygenation should increase the tumor killing efficacy. However, identification of the appropriate timing of each process should not be trivial.

Various ligands towards endothelial molecular targets have been developed and tested mainly for imaging of angiogenesis, but the same ligands can be in principle used for targeting of radionuclides. A systematic review on angiogenesis imaging and also on imaging of other aspects of tumor state (proliferation, apoptosis, hypoxia, etc) is authored by BritzCunningham and Adelstein (2003). An interesting approach for vasculature targeting that has been extensively pursued is to first preactivate the neovasculature so as to increase the density of molecular targets on the endothelial cells and then to deliver the killing radiation or other therapeutics. This approach is reviewed in the following section.

\section{Preactivating}

Upregulation of targeting proteins on the endothelial cells comprising the tumor's blood vessels is the aim of radiation-guided drug delivery using external radiation such as X-rays. At a second step, ligand-conjugated drug carriers are targeted towards the upregulated protein targets of the neovasculature.

For antiangiogenic therapies, high tumor-to-normal organ ratios can be potentially achieved by inducing overexpression of targeting proteins on the tumor neovasculature when these proteins occur on the luminar surface of the endothelium. Characteristic protein examples include the intercellular adhesion molecule-1 (ICAM-1), E-selectrin, P-selectin (Hallahan et al 2001a), and the $\beta_{3}$ integrins (Hallahan et al 2001b).

In particular, Hallahan and colleagues (2003) caused upregulation of the $\alpha_{2 b} \beta_{3}$ receptor of tumor microvasculature using ionizing radiation. As targeting ligand they used fibrinogen - the native ligand of $\alpha_{2 b} \beta_{3}$ - or peptide fragments of fibrinogen containing the RGD motif. The ligand was conjugated to nanoparticles and other delivery carriers such as liposomes which were then administered to embolize the activated tumor microvasculatures in vivo in a murine melanoma animal model. This approach resulted in significant decrease of the tumor blood flow that significantly delayed tumor growth.

Preferential targeting of irradiated tumors resulting in significant delay of tumor growth was also observed in vascularized B16-F10 melanoma xenografts that were initially irradiated by $\mathrm{x}$-rays to increase expression of the alpha $\mathrm{V}$ beta 3 integrin on the tumor endothelium. They were then targeted by liposomes conjugated to RGD sequences with encapsulated combretastatin that is an antivascular agent already evaluated on clinical trials (Pattillo et al 2005).

The strategy of radiation-guided targeted drug delivery to tumor blood cells seems to be promising and has been used till now to deliver conventional therapeutic compounds and radionuclides for imaging (Hallahan et al 2003). Studies on the delivery of therapeutic radionuclides for targeted antiangiogenic internal radiotherapy after upregulation of the molecular targets by external radiation are expected that will soon be reported.

\section{Meta-activating strategies}

By the term "meta-activating", we refer to therapeutic strategies where prodrugs that have been delivered to tumor sites are activated in situ by an external stimulus which could be neutrons, X-rays, gamma photons, or another source of radiation. This type of localized therapy is designed to result in minimal toxicities. This would be achievable as long as the inactive prodrug form that accumulates at normal organs is decomposed and removed from the body with minimal side effects.

In internal radionuclide therapy the most common example is boron neutron capture therapy (BNCT). In this approach, the prodrug is the stable isotope Boron-10 that upon irradiation by thermal neutrons, forms the highly energetic particulate species ${ }^{7} \mathrm{Li}$ and ${ }^{4} \mathrm{He}$ (an alpha particle) by neutron capture and nuclear fission, and a $0.48 \mathrm{MeV}$ gamma photon is emitted via the neutron capture mechanism. In addition, the total dose in tissue has two more components: the gamma ray of energy $2.22 \mathrm{MeV}$ that is released due to thermal neutron capture by hydrogen atoms in tissue, and a proton due to reaction of thermal neutrons with the tissue nitrogen (Ryan and Poston 2005). The dose due to interaction of thermal neutrons with tissues is not insignificant, but lower than the dose due to alpha particles emitted from irradiated ${ }^{10} \mathrm{~B}$.

Because the recoil range of the emitted lithium and helium particles in tissue is 5 and $7 \mu \mathrm{m}$, respectively, which is comparable to the size of a single cell, the cellular internalization and subcellular localization of the boron carrier with respect 
to the cell nucleus is a major determinant of the therapeutic efficacy for this approach. The chemical form of the boron isotope is of great importance for BNCT because it may interfere with boron's subcellular localization (cytoplasmic, exoplasmic, or endonuclear). $\mathrm{pH}$-sensitive drug carriers that become endocytosed and release their contents into the cytoplasm, such as liposomes, could provide a potential answer to the subcellular localization issue. In order to achieve a lethal dose per cancer cell, $10^{9}$ Boron-10 atoms (Javid et al 1952; Tolpin et al 1975; Hartman and Carlsson 1994) need to be delivered per target cell. An informative review by Zamenhof (1997) analyzes the issues related to the short recoil of emitted particles in terms of boron microdosimetric evaluations.

Using monoclonal antibodies serving both as the targeting ligand and the delivery carrier would be challenging for this type of therapy. This is because heavy boron loads are required for adequate delivered doses to cancer cells, and antibody conjugation generally results in relatively low prodrug-to-antibody ratios. Extended conjugation of antibodies for boron loading would not be a solution as it could interfere with the antibody's immunoreactivity potentially resulting in lower tumor accumulation and higher liver uptake (Paxton et al 1992). To address these issues, bispecific antibodies were successfully designed. These antibodies bind to tumor specific antigens and also recognize a hapten included on the boron-containing carborane constructs (Pak et al 1995). For BNCT, other types of carriers, such as dendrimers, and liposomes with high prodrug-to-carrier ratios are also studied, both as targeted and as passive delivery carriers. A disadvantage for neutron capture therapy is the availability since thermal neutron beams originate from nuclear reactors. However, epithermal neutron beams with energy ranging from $0.5 \mathrm{eV}$ to $1 \mathrm{keV}$ can be produced by charged-particle accelerators that can be installed in an urban environment. Epithermal neutrons loose energy while traversing the tissue and become capturable when they reach the boron targets.

\section{Dendrimer-based delivery}

Dendrimers are spherical monodispersed multivalent polymers with high degrees of branching. They have been extensively studied as carriers for the delivery of chemotherapeutics, prodrugs, radionuclides and other molecules for therapy and imaging of cancer (Gillies and Frechet 2005), although their toxicity is still unclear (Duncan and Izzo 2005).

Accumulation of boronated polyamidoamine (PAMAM) dendrimers is significant at the liver and spleen, and seems to be directly related to the dendrimer generation. This is the major challenge using these constructs. In particular, intraperitoneal administration of second and fourth generation of boronated dendrimers with reactive terminal amino groups labeled with tumor specific antibodies were evaluated in tumor-free mice and in mice bearing subcutaneous B16 murine melanoma implants (Barth et al 1994). The reported liver uptake was higher than the accumulation at the tumors at all time points ranging from one to five days. Surface modification of dendrimers to reduce liver uptake will be critical for the successful implementation of these constructs.

In another study, PEGylation was used to increase the blood circulation times of third generation boronated PAMAM dendrimers. In addition, dendrimers were labeled with folic acid to improve their targeting and accumulation to tumor cells overexpressing folate receptors (Shukla et al 2003). However, although in vitro studies showed receptor-mediated uptake, the biodistributions in mice bearing subcutaneous folate receptor (+) murine 24JK-FBP sarcomas confirmed the complexity of using denrimers. Two PEGylated forms of dendrimers were evaluated: the first type contained approximately one PEG chain of $2000 \mathrm{MW}$ per dendrimer with the folic acid attached to its distal end. The second type contained one PEG chain of 2000MW and in addition a shorter PEG chain of $800 \mathrm{MW}$ with the folic acid attached to its distal end. In vivo studies showed that PEGylation reduced the renal uptake that was very high for non-PEGylated conjugates. Hepatic uptake was reduced with diPEGylation. However, in the later case, although the tumor uptake was significant (6.0\% ID/g tumor), the accumulation at the liver, kidney, and spleen was $6.5,10.5$, and 4 times higher than the tumor uptake 6 hours after intraperitoneal administration of the targeted boronated dendrimers. The degree and type of PEGylation was shown to play a critical role on the pharmacokinetics of these systems. The high values of normal organ uptake suggest that further optimization will be required for targeted PEGylated dendrimers to become applicable in vivo.

To bypass the challenge of the circulation times of dendrimers that are shorter than those required for significant accumulation into solid tumors (Malik et al 2000), more accessible molecular targets such as those found on the tumor endothelial walls have been proposed for systemically administered boronated dendrimers. Fifth-generation boronated PAMAM dendrimers (Baker et al 2005) conjugated to a vascular endothelial growth factor (VEGF) targeting the VEGF-2 receptor of the tumor neovasculature were evaluated in a $4 \mathrm{~T} 1$ breast carcinoma mouse model. The in vivo 
studies showed selective accumulation at the areas of active angiogenesis consistent with a VEGFR-2-mediated binding mechanism that was verified in vitro. Application of boronated dendrimers with high prodrug-to-carrier loads to target easily assessable disease may be an appropriate fit for these constructs. The localization of these boronated bioconjugates in endothelial cells, and how their subcellular distributions interfere with their therapeutic efficacy is left to be explored. These boronated bioconjugates may provide an effective approach for antiangiogenic therapy using BNCT.

\section{Liposome-based delivery}

Mostly due to the high encapsulation efficiency of water soluble compounds, several studies have evaluated the use of liposomes in BNCT in vivo.

Liposomes - with and without PEGylation - encapsulating different boron-containing species were studied by Feakes and colleagues (1994), and were evaluated in vivo on a EMT6 tumor bearing mouse model. The rationale for using liposomes, besides the altered biodistributions and pharmacokinetics, is liposomes' ability to encapsulate and deliver free hydrophilic forms of boron to cancer cells. This is of great importance as only those boron forms that are released intracellularly and react with intracellular proteins could be adequately retained by the tumors. If boron retention by tumors is sustained for long time periods, then toxicity to normal organs, as is the liver, could be minimized. In other words, sustained tumor retention would offer the circulating liposomes enough time to clear from the blood and other tissues before activation of the tumor accumulated prodrug. Feakes and colleagues (1994) report for plain non-PEGylated liposomes, sixteen hours after intravenous administration, the tumor-to-blood ratio to be 5 and the boron accumulation at the tumor to be comparable to required therapeutic values for BNCT. Similar tumor-to-blood ratios have been reported by other groups studying liposomal delivery of boronated species (Hawthorne and Shelly 1997). For PEGylated liposomes, Feakes and colleagues (1994) report that the blood circulation time was further extended and after 48 hours the tumor-to-blood ratio was evaluated to have the lower value of 2.4. In these studies, liposomes exhibited tumor uptake and boron retention that were sustained over time. However, liver is a common site of liposome accumulation. In these studies, it exhibited boron uptake comparable with the tumor accumulation values, which, however, were significantly lower than values obtained with boron-conjugated dendrimers as mentioned in the previous paragraphs. To appropriately evaluate the therapeutic efficacy and toxicity of liposome-mediated BNCT, it would be necessary to determine the clearance kinetics of liposomes from normal organs and to optimize the timing for boron activation relative to tumor retention times.

As mentioned above, a key challenge in BNCT is the delivery of adequate prodrug concentrations to cause cell kill upon activation. To achieve high encapsulated boron contents in liposomes both passive loading of boron-containing agents and active loading approaches have been explored. Pan and colleagues (2002b) developed remote-loading protocols of boronated polyamines using a $\mathrm{pH}$-gradient across the liposome membranes. They demonstrated higher loading efficiencies when lower molecular weight boron derivatives were used in liposomes encapsulating ammonium sulfate as the trapping agent. Liposomes were then targeted to the folate receptors of human KB squamous epithelial cancer cells. Neither the chemical form of boron nor the mechanism of boron loading in liposomes did influence the incracellularly accumulated boron that was delivered to cancer cells by targeting liposomes. This study concluded that high concentrations of boron per cell could be delivered that would greatly exceed the required lethal boron amount per cell for effective BNCT. To evaluate feasibility in vivo, the retention times of boron compounds by cancer cells relative to liposome circulation times and clearance from normal organs should be investigated.

In another study, increased tumor accumulation in mice bearing subcutaneous FR (+) M109 murine lung carcinomas was detected for folic-acid labeled PEGylated liposomes with encapsulated boron compounds following intravenous administration. High tumor-to-blood ratio was detected for the targeting liposomes until 72 hours post-administration. However, the tumor uptake of targeting liposomes was comparable to nontargeting liposomes. This result possibly resonates with the mechanism of the enhanced tumor permeability and retention that depends primarily on the size of the delivery carrier. Both carriers were based on the same liposome membrane and size. However, upon extravasation into the tumor interstitium, targeting ligands on the liposome surface may enhance their cell uptake and internalization (Kirpotin et al 2006). Cellular internalization and the subcellular localization of targeting and non-targeting boronated liposomes require further investigation to evaluate their therapeutic effect (Pan et al 2002a). Promising results regarding the delivered prodrug dose per cell have been reported (from in vitro studies) with anti-EGFR liposomes with encapsulated boron (Kullberg et al 2005). Also, RGD-labeled liposomes have also been studied to target the endothelial wall of the tumor neovasculature (Koning et al 2004). 
Transferin labeled PEGylated liposomes with encapsulated boron compounds exhibited tumor suppression and improved long-term survival to subcutaneous colon 26 tumors in mice. Liposomes were administered intravenously. Seventy-two hours after administration, when the tumor-to-blood ratio was 6 and the liver accumulation was less than the tumor uptake, neutron activation took place (Maruyama et al 2004). These liposomes were shown to deliver therapeutic boron doses to the tumors. It was suggested that transferin-labeling enhances retention of liposomes in the tumors and also mediates liposome internalization by cancer cells that constitute the tumors. Internalization and release of encapsulated contents would increase the therapeutic efficacy by allowing boron-conjugates to approach the cell nuclei. Towards the enhancement of intracellular release of contents, liposomes that can directly fuse with the cell membrane and are composed of the positively charged DOTAP and the zwitterionic DOPE (a hexagonal phase lipid) were studied for BNCT (Ristori et al 2005). These compositions are already used in gene therapy, but they will probably require some additional degree of stealthness during blood circulation since their positive charge could result in nonspecific interactions with healthy cells, and in significant uptake by Kupffer cells residing in the liver (Zhang et al 2005).

Other liposome based structures involve boronated lipid (Justus et al 2007) or cholesterol (Thirumamagal et al 2006) derivatives incorporated into the lipid bilayer. These approaches do not take advantage of the encapsulated aqueous compartment.

\section{Other structures}

Other carriers studied for delivery of boron compounds for BNCT include cationic acrylamide copolymers. Copolymers with variable positive charge and mean size of $10 \mathrm{kDa}$ were evaluated for uptake by induced polyps on the luminal side of the gut in rats. The cationic monomer content was varied and boron uptake by the polyps was shown to be an inverse function of the cationic content of the copolymer. This was attributed to the thicker and more negative-richer in sialic acid - mucus that is due to higher mucin production by colon carcinoma cells. The ratios of boron content between the polyps and the surrounding normal epithelium ranged from 6.5 to 1.5 with the higher ratio characterizing the less cationic copolymers (Azab et al 2005). The authors of this study concluded that for residual disease in the colon, BNCT could achieve therapeutic effects with minimal toxicities when delivered and targeted by cationic acrylamide copolymers that will be administered by microenema.
Single-walled carbon nanotubes (SWCNT) have also been studied as carriers of boron for BNCT. Boronated SWCNTs were administered intravenously in mice bearing EMT6 mammary carcinomas. SWCNTs were dissolved and administered in either saline or DMSO solutions. Fast blood clearance was detected, and tumor to blood ratio was 3.12 and 6.13 for saline and DMSO, respectively, 48 hours after administration. Both solutions showed stable tumor retention over a period of 48 hours with tumor-delivered boron doses slightly lower than the therapeutic values. The liver, lung, and spleen uptake was lower than tumor uptake; however, kidney accumulation was not reported. These promising results need to be augmented by elucidation of SWCNTs cell binding and subcellular localization (Yinghuai et al 2005).

\section{Easily accessible disease}

Easily accessible refers to these types of disease for which the drug delivery carriers after administration are in direct contact or at the very close vicinity of the targeted cancer cells. We will review studies on drug carriers that are administered intraperitoneally to directly target intraperitoneally disseminated metastatic cancer. Radioimmunotherapy (RIT) of hematopoietic cancers is also a vast area of research that falls within this category. Included also, are approaches to decrease the toxicity of these therapies to normal organs.

\section{Peritoneal carcinomatosis}

Peritoneal carcinomatosis is common for several metastatic gynecological and gastrointestinal cancers. A promising approach for the therapy of peritoneally disseminated cancer is intraperitoneal administration of therapeutic agents. The rationale for intraperitoneal administration is twofold. First, for micrometastatic tumors in the peritoneal cavity developed vasculature in the peritoneal tumor does not exist ( $\mathrm{Li}$ et al 2000). For these particular cases, intravenous administration of therapeutics will not be the optimal route to target intraperitoneal micrometastases.

Second, since intraperitoneal administration provides direct access to peritoneally disseminated disease, high concentrations of the therapeutic agents could be achieved in the peritoneal cavity, before their concentration reaches toxic levels in the dose limiting organs (Buijs et al 1998; Borchardt et al 2003). Particularly for internal radiotherapy, it is conceivable to match the clearance kinetics from the peritoneum and the kinetics of normal organ uptake with the half-life of the delivered radioisotope so as to minimize irradiation of normal organs. In this way, by the time accumulation of the radiolabeled carrier at normal organs starts 
to become significant, the therapeutic radionuclide can be chosen so as to have mostly decayed. However, in order to achieve therapeutic effects the drug delivery carriers are required to exhibit sustained retention in the peritoneal cavity increasing the probability of encountering tumor sites. Relatively large delivery carriers such as microspheres and liposomes are promising in this sense as they show slow clearance from the peritoneal cavity (Delgado et al 1989; Verschraegen et al 2003).

In peritoneal carcinomatosis, traditional therapeutic procedures may involve peritoneal surgical debulking followed by systemic administration of chemotherapeutics and radiation. Direct intraperitoneal administration has been studied and appears promising against minimal residual disease but not bulky disease (Epenetos et al 1987; Crippa et al 1995; Meredith et al 1996). Alpha particle emitters due to their short range in tissues matching the dimensions of micrometastatic disease could be a logical choice (Meredith et al 1996; Borchardt et al 2003; Palm et al 2007). The high LET of these emitters is advantageous for high killing efficacy. Beta particle emitters would deposit high enough dose only to relatively larger tumors, and could potentially irradiate in significant depth the surrounding healthy tissue.

For locoregional therapy in the peritoneum, several different carriers and targeting systems have been studied for the delivery of radionuclides. One of the first published studies investigated the administration of micron-size colloids labeled with the alpha-emitter Astatine-211 $\left(\mathrm{t}_{1 / 2}=7.21 \mathrm{hr}\right.$, $\mathrm{E}=6.8 \mathrm{MeV}$ and $65 \mu \mathrm{m}$ path length in tissue). Tellurium was chosen to form the colloid microspheres due to its strong affinity for astatine. These studies showed curative effects that were dose depended without serious morbidity in mice with intraperitoneally disseminated murine ovarian carcinoma. They also showed lower toxicity relative to studies where intravenous administration of comparable ${ }^{211}$ At doses were delivered, emphasizing in this way the importance of regional administration (Bloomer et al 1981).

A few characteristic examples of several radionuclide carriers and approaches are given below in more detail covering different strategies that range from radiolabeled monoclonal antibodies to dendrimers, protein complexes, polypeptides, and liposomes in direct (single step) or multistep (pretargeting) schemes.

Borchardt and colleagues (2003) compared the intraperitoneal and intravenous administration of an anti-HER2/neu antibody - trastuzumab- labeled with the alpha particle generator Actinium-225 $\left(\mathrm{t}_{1 / 2}=10\right.$ days, four emitted alpha particles per parent decay) in mice bearing peritoneally disseminated ovarian carcinoma that resembled micrometastatic disease. The study showed significantly enhanced tumor localization of the radionuclides when administered intraperitoneally without increase of normal organ toxicities compared to the intravenous route. To reduce toxicities to normal organs due to released radioactive daughters from the raioimmunoconjugates addition of compounds for faster clearance of radio-toxins have been proposed (Jaggi et al 2005).

Another approach for the reduction of toxicities to normal organs is to decrease the loss of radioactive daughters from the ${ }^{225} \mathrm{Ac}$ carrier by developing large multivesicular antiHER2/neu-liposomes. These are large $650 \mathrm{~nm}$ in diameter liposomes encapsulating smaller lipid vesicles that contain the parent radionuclide. They are proposed for locoregional therapy of peritoneally disseminated ovarian cancer micrometastases. These liposomes are shown to exhibit retention of $20 \%$ of ${ }^{123} \mathrm{Bi}$ - the last alpha emitting radioactive daughter - due to their internal structure. This translates into control of 2.6 out of 4 alpha particles that are generated per ${ }^{225}$ Ac. These anti-HER2/neu-liposomes were shown to exhibit specific binding and internalization by cancer cells in vitro (Sofou et al 2007). At the tumors, in vivo, the delivered activities of ${ }^{225} \mathrm{Ac}$ by liposomes were comparable to those delivered by radiolabeled antibodies twenty four hours after intraperitoneal administration, and showed higher daughter-to-parent ratios compared to the ratios exhibited when antibodies were administered (Sofou et al 2004a).

For the therapy of intraperitoneally disseminated tumors, dendrimers have been studied as delivery carriers for Auger emitters to improve the radionuclide load that is required for therapeutic applications. Multiple chelating agents were attached to biotinylated dendrimers that were mixed with avidin to form larger complexes. Intraperitoneal administration of dendrimer complexes with high ${ }^{111}$ In specific activities was performed on animals bearing intraperitoneal disseminated ovarian cancer tumors (Mamede et al 2003). The cationic charge of the fourth generation dendrimers forming the complexes, combined with the reported ability of avidin to enhance tumor accumulation, resulted in enhanced binding and internalization of the complexes by ovarian carcinoma cells with high tumor accumulation in vivo. In treatment studies, these high dose complexes showed tolerable and dose-depended therapeutic effects.

Multistep or pretargeting approaches have been explored in intraperitoneal radiotherapy but not as extensively as in intravenous RIT (vide infra). For maximum killing efficacy and minimized toxicities, pretargeting strategies aim to decouple the pharmacokinetics of targeting ligands - that may 
be characterized by slow tumor uptake, small binding fraction to tumors, or slow clearance from normal organs-from the potentially different kinetics required for the therapeutic radionuclides. Briefly, at the first step, a tumor-targeting molecule such as an antibody is administered. This targeting molecule should have high affinity for cancer cells, is not radiolabeled, and also bears a second functionality that serves as a linker for the targeting in the following second step. Only after the fist unlabeled bifunctional antibody is adequately cleared from the body but not from the tumor sites, a radiolabeled "effector" compound is administered. The effector compound is designed to bind with very high avidity to the second functionality of the bifunctional antibody of the first targeting step. The radiolabeled effector compound should have faster pharmacokinetics to clear rapidly from circulation and the body, and could potentially also exhibit better permeation in the tumor.

To target ${ }^{211} \mathrm{At}$ to intraperitoneal tumors, Lindegren and colleagues (2003) used a pretargeting compound containing avidin as the second functionality. At the second step, the effector molecule polylysine was introduced in a biotinylated form and was used as a multicarrier for ${ }^{211} \mathrm{At}$. The rationale for this approach is the potentially high specific radioactivity of polylysine that would enable delivery of high radiation doses for therapy. Biotinylated polylysines of various sizes exhibited strong binding to avidin-coated beads. Following intraperitoneal administration of the effector molecule, it was found that increasing molecular weights shifted the normal organ accumulation from the kidneys to the liver. Increased uptake at the thyroid, lungs, stomach, and the spleen was detected following administration of effector molecules ( 38 and $363 \mathrm{kDa}$ ) (Lindegren et al 2002). The authors suggest that this is caused by possible degradation of the polymers by the liver resulting in release of the radionuclide as free astatine/astatide. Addressing the issue of liver accumulation and radiolabel release would significantly advance this promising approach.

Beta emitters with short range in tissue such as ${ }^{177} \mathrm{Lu}$ $\left(\mathrm{t}_{1 / 2}=6.61\right.$ days, $\mathrm{E}_{\max }=497 \mathrm{keV}$ for beta emissions) have been proposed as alternatives for targeting intraperitoneal carcinomatosis. Lutetium-177 emits beta particles with average penetration in soft tissues ranging from 0.2 to 0.3 $\mathrm{mm}$. Two beta particle emitters, ${ }^{90} \mathrm{Y}$ and ${ }^{177} \mathrm{Lu}$, were studied and compared in a pretargeting approach for intraperitoneal therapy. Yttium-90's emitted beta particles travel as far as $2.76 \mathrm{~mm}$ in tissue. Lutitium-177 in addition to beta particles emits low energy gamma radiation of 113 and 208 $\mathrm{keV}$ allowing for gamma camera imaging. In this study, a tetrameric fusion protein composed of CC49-scFv-chains on streptavidin was initially administered intraperitoneally to mice bearing peritoneal TAG-71-positive LS174T tumors. The CC49 protein was chosen based on its good tumor uptake and on evidence of no localization to normal gastrointestinal tissues. To reduce bone marrow suppression, the blood circulating non bound fusion protein was cleared from circulation by intravenous administration of a synthetic clearing agent having high affinity for hepatocytes for rapid removal from the blood, and fast internalization and catabolism by the liver cells. Then radionuclide-DOTA-biotin was administered intraperitoneally. In therapeutic studies, the effector compound ${ }^{177} \mathrm{Lu}$-DOTA-biotin produced prolonged survival and appeared to be less toxic than ${ }^{90} \mathrm{Y}$-DOTA-biotin. At the maximum dose tested of $800 \mu \mathrm{Ci}, 60 \%$ of deaths were early deaths due to toxicity of ${ }^{90} \mathrm{Y}$-DOTA-biotin. No evidence of toxicity was observed for ${ }^{177} \mathrm{Lu}$-DOTA-biotin at the same dose. This result was attributed to the shorter recoil range of the ${ }^{177} \mathrm{Lu}$ emitted beta particles (Buchsbaum et al 2005b).

\section{Radioimmunotherapy}

In Radioimmunotherapy (RIT), tumor-targeting antibodies are utilized for the delivery of therapeutic radionuclides. RIT is emerging as a very promising therapeutic modality, and progressively gains more ground compared with traditional chemotherapy. Although treatment of solid tumors is still a challenge for RIT mainly due to limited tumor cell accessibility by circulating antibodies (Jhanwar and Divgi 2005; Sharkey and Goldenberg 2005), RIT has been successful with small volume disease or minimal residual disease particularly with hematopoietic neoplasms (Sharkey and Goldenberg 2005). The two main components in RIT are the type of antibody used and the therapeutic radionuclides. For a systematic analysis of the existing approaches the readers are referred to reviews by Goldenberg (Goldenberg 2002) and Sgouros (Sgouros 2002). Although most RIT trials and commercial radioimmunoconstructs involve beta particle emitters, clinical RIT trials with alpha particle emitters on small volume disease have demonstrated safety, feasibility and activity against cancer cells (Mulford et al 2005), and more alpha particle emitting isotopes are increasingly studied.

\section{Single-step RIT}

In single-step RIT, an initial major challenge was the undesirable immunogenicity of the administrated antibodies that could prevent repeated therapeutic cycles. Advances in antibody engineering have been employed to provide antibodies with decreased immunogenic responses and antibody 
fragments. However, smaller radioimmunoconjugates exhibit different biodistributions that essentially shift toxicities to other organs. As the antibody size decreases, toxicities shift from the bone marrow and the liver to the kidneys. In addition, the tumor binding uptake and retention of smaller fragments are decreased compared to the complete IgG's. The different kinetic profiles of smaller radioimmunoconjugates can be successfully addressed by choosing radionuclides with matching half-lives, but the lower tumor accumulation observed for smaller antibody fragments may require higher administered doses to achieve lethal absorbed doses at the targeted cancer cells. This could proportionally increase toxicity at critical organs (Goldenberg 2002).

\section{Multistep targeting}

Multistep targeting is proposed as an alternative to combine reduced toxicities at normal organs with increased tumorto-normal organ ratios, and eventually deliver higher doses of radiation at the tumor. Numerous constructs, including monoand bi-functional compounds and radionuclide combinations have been designed and evaluated. For extensive overviews on preclinical and clinical studies on RIT the readers are referred to other reviews specific on antibody pretargeting for radioimmunotherapy (Bethge and Sandmaier 2005; Koppe et al 2005; Sharkey and Goldenberg 2005; Goldenberg et al 2006; Pohlman et al 2006).

The radiolabeling efficiency - specific activity - of the construct that is used at the final targeting step plays a determining role on the delivered radiation dose at the tumor. Several carriers other than antibodies, such as polypeptides and dendrimers that can be heavily radiolabeled, have been studied to improve the efficacy of this final step. However, the accumulation of these constructs to normal organs usually renders these approaches questionable for effective therapy (del Rosario and Wahl 1993; Wilbur et al 1998). For example, starburst PAMAM polyamidoamine dendrimers have been reported for the targeted delivery of ${ }^{111} \mathrm{In}$ and ${ }^{88} \mathrm{Y}\left(\mathrm{t}_{1 / 2}=106.65\right.$ days, beta emissions with $\mathrm{E}_{\max }=0.76 \mathrm{MeV}$, gamma photons of $\mathrm{E}=1.83 \mathrm{MeV}$ ). Second generation dendrimers are highly branched and are proposed as alternative chelating platforms for the attachment of radionuclides (Kobayashi et al 1999). However, the positively charged dendrimers seem to extensively accumulate in the liver, spleen, and the pancreas. Alternative approaches to address this toxicity issue are necessary.

A different approach to multistep targeting is MitraDep ${ }^{\circledR}$ that aims to decrease toxicity at normal organs while maintaining high administered radiation doses for high tumor delivered doses. This approach involves the direct clearing of the circulating radiolabeled antibodies from the blood by extracorporeal depletion at the time point when their tumor localization becomes adequate and before the accumulation in normal organs becomes significant. This is achieved by passing the entire blood flow through a filter coated with avidin to remove the radiolabeled antibodies that have been simultaneously biotinylated. The antibody's functionalization takes place through the trifunctional chelator [3-(13'-thioureabenzyl-DOTA)trioxadiamine-1-(13' '-biotin-Asp-OH)trioxadiamine-5-isothiocyanato-aminoisophtalate] to ensure the ratio of biotin-to-DOTA is 1:1. Otherwise, the radiolabeled antibodies that are not biotinylated would not be cleared using extracorporeal depletion. The reported phase I study, shows that this procedure is safe in the sense that it does not expose the patients to risks such as blood coagulation, perturbation of hematological parameters, activation of the immunological system or decrease of electrolytes in the blood. In addition, for ${ }^{111}$ In labeled rituximab that was used as ${ }^{90} \mathrm{Y}$ surrogate, $62 \%$ depletion of activity was detected in the lungs, and 40\% in the liver and kidneys. This device is currently on a dose-escalating phase I/II study (Linden et al 2005).

\section{Preactivated RIT}

As mentioned above, RIT is not as effective for solid tumors as for easily accessible disease. This is because RIT is usually designed to target accessible molecular markers on the surface of tumor cells. For solid tumors, on the contrary, the radioimmuno-constructs need to first extravasate into the tumor interstitium before reaching the tumor cells. Thus, issues related to poor tumor penetration, or low tumor antigen and receptor expression may present significant challenges. To enhance targeting to molecular markers on tumor cells, upregulation of tumor-related antigens and receptors has been proposed using viral vectors or by the administration of cytokines (Rogers et al 2002).

A very interesting example is the upregulation of the somatostatin receptor SSTr2 on the surface of human nonsmall cell lung tumor xenografts before administration of a therapeutic radiolabeled antibody. Following intratumoral administration of an adenovirus encoding the SSTr2 gene, the authors showed that high levels of the somatostatin receptor SSTr2 were induced on the surface of cancer cells. Then, following intravenous administration, these cancer cells were targeted by an ostreotide analogue labeled with the beta-emitter ${ }^{90} \mathrm{Y}$ (Rogers et al 2002). This study showed that upregulation of the targeting receptors on the surface 
of cancer cells comprising the solid tumors is possible to achieve therapeutic efficacy by radiolabeled antibodies with beta emitters that otherwise is not achievable. Somatostatin receptors have also been studied on ovarian cancer xenografts (Rogers et al 1999), and have been upregulated by ionizing radiation (Buchsbaum et al 2005a). The curative potential of ${ }^{90} \mathrm{Y}$-based radiotherapy targeted to somatostatin expressing tumors has been shown in several studies (Stolz et al 1998; de Jong et al 2001). The approach of preactivation could provide a feasible answer to the radioimmunotherapy of solid tumors. Hopefully, current limitations in adenoviral vectors due to high liver toxicities would be eventually surpassed leading to a new direction for RIT.

\section{Conclusions}

In internal radiotherapy of cancer, a variety of diverse parameters characterizing the tumor topology, morphology, and physiology need to be considered for choosing the optimal targeting ligand, delivery carrier, and type of therapeutic radionuclide for effective therapy. In addition, the stability of constructs, and the fate of free radionuclides in vivo play a significant role on the success of the therapeutic strategy. These factors result in the design of different materials and constructs that are briefly summarized in this review.

Currently, clinical trials on targeted radionuclide therapies are mostly based on small molecules both for targeting and delivery that include antibodies, smaller peptides (Wong 2006; Dearling and Pedley 2007), or the radiolabeled biotin/ avidin pair (Paganelli et al 2007). Advancement in the area of internal radionuclide therapy may further be enabled by using different carrier materials with higher radionuclide loads exhibiting different behavior in vivo such as liposomes, dendrimers, and other structures with sizes of the order of several nanometers. Better understanding of the interactions of new constructs with the biological milieu, proof of the safety of new constructs, improvements in radiochemistry for stable and efficient radiolabeling, progress in antibody and other ligand engineering and wide availability of a variety of radionuclides, should enable internal radiotherapy to reach its full potential.

\section{References}

Adam MJ, Wilbur DS. 2005. Radiohalogens for imaging and therapy. Chem Soc Rev, 34:153-63.

Adams GP, Schier R, McCall AM, et al. 2001. High affinity restricts the localization and tumor penetration of single-chain Fv antibody molecules. Cancer Res, 61:4750-5.

Alessi P, Ebbinghaus C, Neri D. 2004. Molecular targeting of angiogenesis. Biochim Biophys Acta, 1654:39-49.
Allen B, Raja C, Rizvi S, et al. 2007. Tumour anti-vascular alpha therapy: a mechanism for the regression of solid tumours in metastatic cancer. Phys Med Biol, 52:L15-9.

Allen C, Dos Santos N, Gallagher R, et al. 2002. Controlling the physical behavior and biological performance of liposome formulations through use of surface grafted poly(ethylene glycol). Bioscience Rep, 22:225-50.

Allen TM. 2002. Ligand-targeted therapeutics in anticancer therapy. Nature Cancer Rev, 2:750-63.

Auguste DT, Prud'homme RK, Ahl PL, et al. 2003. Association of hydrophobically-modified poly(ethylene glycol) with fusogenic liposomes. Biochim Biophys Acta, 1616:184-95.

Azab A-K, Srebnik M, Doviner V, et al. 2005. Targeting normal and neoplastic tissues in the rat jejunum and colon with boronated, cationic acrylamide copolymers. J Control Release, 106:14-25.

Baker MV, Gaynutdinov TI, Patel V, et al. 2005. Vascular endothelial growth factor selectively targets boronated dendrimers to tumor vasculature. Mol Cancer Ther, 4:1423-9.

Barth RF, Adams DM, Soloway AH, et al. 1994. Boronated starburst dendrimer-monoclonal antibody imunoconjugates: evaluation as a potential delivery system for neutron capture therapy. Bioconjugate Chem, 5:58-66.

Bethge WA and Sandmaier BM. 2005. Targeted cancer therapy using radiolabeled monoclonal antibodies. Technol Cancer Res Treat, 4:393-405.

Bloomer WD, McLaughlin WH, Neirinckx RD, et al. 1981. Astatine211 -tellurium radiocolloid cures experimental malignant ascites. Science, 212:340-1.

Borchardt P, Yuan R, Miederer M, et al. 2003. Targeted actinium-225 in vivo generators for therapy of ovarian cancer. Cancer Res, 63:5084.

Boswell CA and Brechbiel MW. 2005. Auger electrons: lethal, low energy, and coming soon to a tumor cell nucleus near you. J Nucl Med, 46:1946-7.

Bottaro DP and Liotta LA. 2003. Cancer: Out of air is not out of action. Nature, 423:593-5.

Brechbiel MW. 2007. Bifunctional chelates for metal nuclides. $Q \mathrm{~J} \mathrm{Nucl}$ Med Mol Imaging, Nov 28 [Epub ahead of print].

Britz-Cunningham SH, Adelstein SJ. 2003. Molecular targeting with radionuclides: state of the science. J Nucl Med, 44:1945-61.

Brooks PC, Clark RA, Cheresh DA. 1994. Requirement of vascular integrin alpha v beta 3 for angiogenesis. Science, 264:569-71.

Bruera E, Neumann CM, Pituskin E, et al. 1999. A randomized controlled trial of local injections of hyaluronidase versus placebo in cancer patients receiving subcutaneous hydration. Ann Oncol, 10:1255-8.

Buchsbaum DJ, Chaudhuri TR, Zinn KR. 2005a. Radiotargeted gene therapy. J Nucl Med, 46:179s-86s.

Buchsbaum DJ, Khazaeli M, Axworthy DB, et al. 2005b. Intraperitoneal pretarget radioimmunotherapy with $\mathrm{CC} 49$ fusion protein. Clin Cancer Res, 15:8180-5.

Buijs WCAM, Tibben JG, Boerman OC, et al. 1998. Dosimetric analysis of chimeric monoclonal antibody cMOv18 $\mathrm{IgG}$ in ovarian carcinoma patients after intraperitoneal and intravenous administration. Eur J Nucl Med, 25:1552-61.

Burg MA, Pasqualini R, Arap W, et al. 1999. NG2 proteoglycan-binding peptides target tumor neovasculature. Cancer Res, 59:2869-74.

Burrows FJ, Thorpe PE. 1993. Eradication of large solid tumors in mice with an immunotoxin directed against tumor vasculature. Proc Natl Acad Sci USA. 90:8996-9000.

Campbell AM, Bailey IH, Burton MA. 2000. Analysis of the distribution of intra-arterial microspheres in human liver following hepatic yttrium-90 microsphere therapy. Phys Med Biol, 45:1023-33.

Carmeliet P, Jain RK. 2000. Angiogenesis in cancer and other diseases. Nature, 407:249-57.

Chunfu Z, Jinquan C, Duanzhi Y, et al. 2004. Preparation and radiolabeling of human serum albumin (HSA)-coated magnetite nanoparticles for magnetically targeted therapy. Appl Radiat Isot, 61:1255-9.

Couturier O, Supiot O, Degraef-Mougin M, et al. 2005. Cancer radioimmunotherapy with alpha-emitting nuclides. Eur J Nucl Med Imaging, 32:601-14. 
Crippa F, Bolis G, Seregni E, et al. 1995. Single-dose intraperitoneal radioimmunotherapy with the murine monoclonal antibody I-131 MOv18: Clinical results in patients with minimal residual disease of ovarian cancer. Eur J Cancer, 31:686-90.

Davies CL, Lundstrom LM, Frengen J, et al. 2004. Radiation improves the distribution and uptake of liposomal Doxorubicin (Caelyx) in human osteosarcoma xenografts. Cancer Res, 64:547-53.

de Jong M, Breeman WAP, Bernard BF, et al. 2001. Tumor response after [90Y-DOTA0,Tyr3]Octreotide radionuclide therapy in a transplantable rat tumor model is dependent on tumor size. J Nucl Med, 42:1841-6.

Dearling JLJ, Pedley RB. 2007. Technological advances in radioimmunotherapy. Clin Oncol, 19:457-69.

del Rosario RB, Wahl RL. 1993. Biotinylated iodo-polylysine for pretargeted radiation delivery. $J$ Nucl Med, 34:1147-51.

Delgado G, Potkul R, Treat J, et al. 1989. A phase I/II study of intraperitoneally administered doxorubicin entrapped. Am J of Obs Gyn, 160:812.

Du J, Marquez M, Hiltunen J, et al. 2000. Radiolabeling of dextran with rhenium-188. Appl Radiat Isot, 53:443-8.

Duncan R, Izzo L. 2005. Dendrimer biocompatibility and toxicity. $A d v$ Drug Deliv Rev, 57:2215-37.

Eikenes L, Bruland OS, Brekken C, et al. 2004. Collagenase increases the transcapillary pressure gradient and improves the uptake and distribution of monoclonal antibodies in human osteosarcoma xenografts Cancer Res, 64:4768-73.

Eikenes L, Tari M, Tufto I, et al. 2005. Hyaluronidase induces a transcapillary pressure gradient and improves the distribution and uptake of liposomal doxorubicin (CaelyxTM) in human osteosarcoma xenografts. Br J Cancer, 93:81-8.

Emfietzoglou D, Kostarelos K, Papakostas A, et al. 2005. Liposomemediated radiotherapeutics within avascular tumor spheroids: comparative dosimetry study for various radionuclides, liposome systems, and a targeting antibody. J Nucl Med, 46:89-97.

Epenetos A, Munro A, Stewart S, et al. 1987. Antibody-guided irradiation of advanced ovarian cancer with intraperitoneally administered radiolabeled monoclonal antibodies. J Clin Oncol, 5:1890-9.

Faraggi G, Gardin I, de Labriolle-Vaylet C, et al. 1994. The influence of tracer localization on the electron dose rate delivered to the cell nucleus. J Nucl Med, 35:113-19.

Feakes DA, Shelly K, Knobler CB, et al. 1994. Na3[B20H17NH3]: Synthesis and liposomal delivery to murine tumors. Proc Natl Acad Sci USA, 91:3029-33.

Folkman J. 1971. Tumor angiogenesis: therapeutic implications. $N$ Engl $J$ Med, 285:1182-6.

Folkman J. 1990. What is the evidence that tumors are angiogenesis dependent? J Natl Cancer Inst, 82:4-6.

Gabizon A, Shmeeda H, Barenholz Y. 2003. Pharmacokinetics of pegylated liposomal Doxorubicin: review of animal and human studies. Clin Pharmacokinet, 42:419-36.

Gillies ER, Frechet JMJ. 2005. Dendrimers and dendritic polymers in drug delivery. Drug Discov Today, 10:35-43.

Goldenberg DM. 2002. Targeted therapy of cancer with radiolabeled antibodies. J Nucl Med, 43:693-713.

Goldenberg DM, Sharkey RM, Paganelli G, et al. 2006. Antibody pretargeting advances cancer radioimmunodetection and radioimmunotherapy. J Clin Oncol, 24:823-34.

Haefeli UO, Sweeney SM, Beresford BA, et al. 1995. Effective targeting of magnetic radioactive Yttrium-90 microspheres to tumor cells by an externally applied magnetic field. Preliminary in vitro and in vivo results. Nucl Med Biol, 22:147-55.

Haefeli UO, Casillas S, Dietz DW, et al. 1999. Hepatic tumor radioembolization in a rat model using radioactive rhenium (186Re/188Re) glass microspheres. Int J Radiation Oncology Biol Phys, 44:189-99.

Haefeli UO, Pauer G, Failing S, et al. 2001. Radiolabeling of magnetic particle with rhenium-188 for cancer therapy. J Magn Magn Mater, 225:73-8.

Hallahan D, Shimian Q, Geng L, et al. 2001a. Radiation-mediated control of drug delivery. Am J Clin Oncol, 24:473-80.
Hallahan D, Geng L, Qu S, et al. 2003. Integrin-mediated targeting of drug delivery to irradiated tumor blood vessels. Cancer Cell, 3:63-74.

Hallahan DE, Geng L, Cmelak A, et al. 2001b. Targeting drug delivery to radiation-induced neoantigens in tumor microvasculature. $J$ Control Release, 74:183-91.

Hartman T, Carlsson J. 1994. Radiation dose heterogeneity in receptor and antigen mediated boron neutron capture therapy. Radiother Oncol, 31:61-75.

Hawthorne MF, Shelly K. 1997. Liposomes as drug delivery vehicles for boron agents. J Neurooncol, 33:53-8.

Hei TK, Persaud R, Zhou H, et al. 2004. Genotoxicity in the eyes of bystander cells. Mutat Res, 568:111-20.

Henriksen G, Bruland OS, Larsen RH. 2004a. Thorium- and Actinium polyphosphate compounds as bone-seeking alpha particle emitting agents. Anticancer Res, 24:101-5.

Henriksen G, Schoultz BW, Michaelsen TE, et al. 2004b. Sterically stabilized liposomes as a carrier for alpha-emitting radium and actinium radionuclides. Nucl Med Biol, 31:441-9.

Hobbs SK, Monsky WL, Yuan F, et al. 1998. Regulation of transport pathways in tumor vessels: Role of tumor type and microenvironment. PNAS, 95:4607-12.

Hobson B, Denekamp J. 1984. Endothelial proliferation in tumours and normal tissues: continuous labelling studies. Br J Cancer, 49:405-13.

Houle S, Yip T-CK, Shepherd FA, et al. 1989. Hepatocellular carcinoma: pilot trial of treatment with Yttrium-90 microspheres. Radiology, $172: 857-60$

Humm JL. 1987. A microdosimetric model of astatine-211 labeled antibodies for radioimmunotherapy. Int J Radiat Oncol Biol Phys, 13:1767-73.

Humm JL, Chin LM. 1993. A model of cell inactivation by alpha-particle internal emitters. Radiat Res, 134:143-50.

Hwang KJ, Merriam JE, Beaumier PL, et al. 1982. Encapsulation, with high efficiency, of radioactive metal ions in liposomes. Biochim Biophys Acta, 716:101-9.

Hynes NE, Lane HA. 2005. ERBB receptors and cancer: the complexity of targeted inhibitors. Nature Rev Cancer, 5:341-54.

Jaggi JS, Kappel BJ, McDevitt MR, et al. 2005. Efforts to control the errent products of a targeted in vivo generator. Cancer Res, 65:4888-95.

Jain RK, Munn LL, Fukumura D. 2002. Dissecting tumor pathophysiology using intravital microscopy. Nature Rev Cancer, 2:266-76.

Jain RK. 2005. Normalization of tumor vasculature: an emerging concept in antiangiogenic therapy. Science, 307:58-62.

Janssen M, Oyen W, Dijkgraaf I, et al. 2002. Tumor targeting with radiolabeled alpha v beta 3 integrin binding peptides in a nude mouse model. Cancer Res, 62:6146-51.

Janssen M, Frielink C, Dijkgraaf I, et al. 2004. Improved tumor targeting of radiolabeled RGD peptides using rapid dose fractionation. Cancer Biother Radiopharm, 19:399-404.

Javid M, Brownell GL, Sweet WH. 1952. The possible use of neutron capture isotopes such as boron-10 in the treatment of neoplasms: II. computation of the radiation energy and estimates of effects in normal and neoplastic brain. $J$ Clin Invest, 31:603-10.

Jhanwar YS, Divgi C. 2005. Current status of therapy of solid tumors. J Nucl Med, 46:141s-50s.

Jonasdottir T, Fisher D, Borrebaek J, et al. 2006. First in vivo evaluation of liposome-encapsulated 223Ra as a potential alpha-particle-emitting cancer therapeutic agent. Anticancer Res, 26:2841-8.

Justus E, Awad D, Hohnholt M, et al. 2007. Synthesis, liposomal preparation, and in vitro toxicity of two novel dodecaborate cluster lipids for boron neutron capture therapy. Bioconjugate Chem, 18:1287-93.

Kalofonos H, Rowlinson G, Epenetos A. 1990. Enhancement of monoclonal antibody uptake in human colon tumor xenografts following irradiation. Cancer Res, 50:159-63.

Kassis AI, Sastry KSR, Adelstein SJ. 1987. Kinetics of uptake, retention, and radiotoxicity of I-125 UDR in mammalian cells: implications of localized energy deposition by Auger processes. Radiat Res, 109:78-89.

Kassis AI. 2004. The amazing world of Auger electrons. Int J Radiat Biol, 80:789-803. 
Kassis AI, Adelstein SJ. 2005. Radiobiologic principles in radionuclide therapy. J Nucl Med, 46:4s-12s.

Kennel SJ, Mirzadeh S. 1998. Vascular targeted radioimmunotherapy with 213Bi - an alpha-particle emitter. Nucl Med Biol, 25:241-6.

Kirpotin DB, Drummond DC, Shao Y, et al. 2006. Antibody targeting of long-circulating lipidic nanoparticles does not increase tumor localization but does increase internalization in animal models. Cancer Res, 66:6732-40.

Kobayashi H, Wu C, Kim M-K, et al. 1999. Evaluation of the in vivo evaluation of indium-111 and yttrium-88 labeled dendrimer-1B4M-DTPA and its conjugation with anti-Tac monoclonal antibody. Bioconjugate Chem, 10:103-11.

Koivunen E, Wang B, Ruoslahti E. 1995. Phage libraries displaying cyclic peptides with different ring sizes: ligand specificities of the RDGdirected integrins. Biotechnology, 13:265-70.

Kolbert KS, Hamacher KA, Jurcic JG, et al. 2001. Parametric Images of Antibody Pharmacokinetics in Bi213-HuM195 Therapy of Leukemia. J Nucl Med, 42:27-32.

Kolbert KS, Watson T, Matei C, et al. 2003. Murine S factors for liver, spleen, and kidney. $J$ Nucl Med, 44:784-91.

Koning GA, Fretz MM, Woroniecka U, et al. 2004. Targeting liposomes to tumor endothelial cells for neutron capture therapy. Appl Radiat Isot, 61:963-7.

Koppe MJ, Postema EJ, Aarts F, et al. 2005. Antibody-guided radiation therapy of cancer. Cancer Metastasis Rev, 24:539-67.

Kullberg EB, Wei Q, Capala J, et al. 2005. EGF-receptor targeted liposomes with boronated acridine: growth inhibition of cultured glioma cells after neutron irradiation. Int J Radiat Biol, 81:621-9.

Kvols LK. 2005. Radiation sensitizers: a selective review of molecules targeting DNA and non-DNA targets. $J$ Nucl Med, 46:187s-90s.

Lasic DD. 1993 Liposomes from Physics to Applications. Amsterdam, Elsevier.

Lasic DD, Martin F (eds). 1995. Stealth liposomes, Boca Raton, Fla, CRC Pr.

Li C-Y, Shan S, Huang Q, et al. 2000. Initial stages of tumor cell-induced angiogenesis: Evaluation via skin window chambers in rodent models. J Natl Cancer Inst, 92:143-7.

Li J, Huang S, Armstrong EA, et al. 2005. Angiogenesis and radiation response modulation after vascular endothelial growth factor receptor-2 (VEGF-2) blockade. int J Radiat Oncology Biol Phys, 62:1477-85.

Lin G, Lunderquist A, Hagerstrand I, et al. 1984. Postmortem examination of the blood supply and vascular pattern of small liver metastases in man. Surgery, 96:517-26.

Lindegren S, Andersson H, Jacobsson L, et al. 2002. Synthesis and biodistribution of 211 At-labeled, biotinylated, and charge-modified poly-L-lysine: evaluation for use as an effector molecule in pretargeted intraperitoneal tumor therapy. Bioconjugate Chem, 13:502-9.

Lindegren S, Karlsson B, Jacobsson L, et al. 2003. 211At-labeled and biotinylated effector molecules for pretargeted radioimmunotherapy using poly-L- and poly-D-lysine as multicarriers. Clin Cancer Res, 9:3873s-9.

Linden O, Kurkus J, Garkavij M, et al. 2005. A novel platform for radioimmunotherapy: extracorporeal depletion of biotinylated and 90Y-labeled Rituximab in patients with refractory B-cell lymphoma. Cancer Biother Radiopharm, 20:457-66.

Line BR, Mitra A, Nan A, et al. 2005. Targeting tumor angiogenesis: comparison of peptide and polymer-peptide conjugates. $\mathrm{J} \mathrm{Nucl} \mathrm{Med,}$ 46:1552-60.

Macklis RM, Kinsey BM, Kassis AI, et al. 1988. Radioimmunotherapy with alpha-particle-emitting immunoconjugates. Science, 240:1024-6.

Maeda H, Wu J, Sawa T, et al. 2000. Tumor vascular permeability and the EPR effect in macromolecular therapeutics: a review. $J$ Control Release, 65:271-84.

Malik N, Wiwattanapatapee R, Klopsch R, et al. 2000. Relationship between structure and biocompatibility in vitro, and preliminary studies on the biodistribution of 125I-labelled polyamidoamine dendrimers in vivo J Control Release, 65:133-48.
Mamede M, Saga T, Kobayashi H, et al. 2003. Radiolabeling of avidin with very high specific activity for internal radiation therapy of intraperitoneal disseminated tumors. Clin Cancer Res, 9:3756-62.

Mantravadi RVP, Spigos DG, Tan WS, et al. 1982. Intraarterial Yttrium-90 in the treatment of hepatic malignancy. Radiolgy, 142:783-6.

Maruyama K, Ishida O, Kasaoka S, et al. 2004. Intracellular targeting of sodium mercaptoundecahydrododecaborate (BSH) to solid tumors by transferrin-PEG liposomes, for boron neutron-capture therapy (BNCT). $J$ Control Release, 98:195-207.

McDevitt MR, Ma D, Lai LT, et al. 2001. Tumor therapy with targeted atomic nanogenerators. Science, 294:1537-40.

Meredith RF, Partridge EE, Alvarez RD, et al. 1996. Intraperitoneal radioimmunotherapy of ovarian cancer with lutetium-177-CC49. J Nucl Med, 37:1491-6.

Mitra A, Nan A, Papadimitriou JC, et al. 2006. Polymer-peptide conjugates for angiogenesis targeted tumor radiotherapy. Nucl Med Biol, 33:43-52.

Mulford DA, Scheinberg DA, Jurcic JG. 2005. The promise of targeted alpha-particle therapy. J Nucl Med, 46:199s-204s.

Murthy R, Nunez R, Szklaruk J, et al. 2005. Yttrium-90 microsphere therapy for hepatic malignancy: Devices, indications, technical considerations, and potential complications. Radiographics \%R 101148/rg25si055515, 25:S41-55.

Netti PA, Berk DA, Swartz MA, et al. 2000. Role of extracellular matrix assembly in interstitial transport in solid tumors. Cancer Res, 60:2497-503.

Nieder C, Wiedenmann N, Andratschke N, et al. 2006. Current status of angiogenesis inhibitors combined with radiation therapy. Cancer Treat Rev, 32:348-64.

Nikula TK, McDevitt MR, Finn RD, et al. 1999. Alpha-emitting bismuth cyclohexylbenzyl DTPA constructs of recombinant humanized antiCD33 antibodies: pharmacokinetics, bioactivity, toxicity and chemistry. J Nucl Med, 40:166-76.

Noguchi Y, Wu J, Duncan R, et al. 1998. Early phase tumor accumulation of macromolecules: A great difference between the tumor vs.normal tissue in their clearance rate. Jpn J Cancer Res, 89:307-14.

Normanno N, De Luca A, Bianco C, et al. 2006. Epidermal growth factor receptor (EGFR) signaling in cancer. Gene, 366:2-16.

Nunez MI, Villalobos M, Olea N, et al. 1995. Radiation-induced DNA double-strand break rejoining in human tumour cells. $\mathrm{Br} J$ Cancer, 71:311-16.

Paganelli G, Ferrari M, Cremonesi M, et al. 2007. IART(R): Intraoperative avidination for radionuclide treatment. A new way of partial breast irradiation. The Breast, 16:17-26.

Pak RH, Primus FJ, Rickard-Dickson KJ, et al. 1995. Preparation and properties of nido-carborane-specific monoclonal antibodies for potential use in boron neutron capture therapy for cancer. Proc Natl Acad Sci, 92:6986-90

Palm S, Enmon R, Scheinberg DA, et al. 2002. Modeling alpha-emitter labeled antiPSMA antibody (225Ac-J591) targeting of prostate micrometastases: microdosimetric analysis of spheroid response. Cancer Biother Radiopharm, 17:488.

Palm S, Back T, Claesson I, et al. 2007. Therapeutic efficacy of astatine211-labeled trastuzumab on radioresistant SKOV-3 tumors in nude mice. Int J Radiat Oncol Biol Phys, 69:572-9.

Pan XQ, Wang H, Lee RJ. 2002a. Boron delivery to a murine lung carcinoma using folate receptor-targeted liposomes. Anticancer Res, 22:1629-33.

Pan XQ, Wang H, Shukla S, et al. 2002b. Boron-containing folate receptor-targeted liposomes as potential delivery agents for neutron capture therapy. Bioconjugate Chem, 13:435-42.

Pattillo CB, Sari-Sarraf F, Nallamothu R, et al. 2005. Targeting of the antivascular drug combretastatin to irradiated tumors results in tumor growth delay. Pharm Res, 22:1117-20.

Paxton RJ, Beatty BG, Varadarajan A, et al. 1992. Carboranyl peptide-antibody conjugates for neutron-capture therapy: preparation, characterization, and in vivo evaluation. Bioconjug Chem, 3:241-7. 
Pohlman B, Sweetenham J, Macklis RM. 2006. Review of clinical radioimmunotherapy. Expert Rev Anticancer Ther, 6:445-61.

Reubi JC, Maecke HR, Krenning EP. 2005. Candidates for peptide receptor

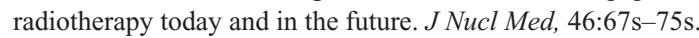

Ristori S, Oberdisse J, Grillo I, et al. 2005. Structural characterization of cationic liposomes loaded with sugar-based carboranes. Biophys $J$, 88:535-47.

Rogers BE, McLean SF, Kirkman RL, et al. 1999. In vivo localization of [111In]-DTPA-D-Phe -Octreotide to human ovarian tumor xenografts induced to express the somatostatin receptor subtype 2 using an adenoviral vector. Clin Cancer Res, 5:383-93.

Rogers BE, Zinn KR, Lin CY, et al. 2002. Targeted radiotherapy with [(90)Y]-SMT 487 in mice bearing human nonsmall cell lung tumor xenografts induced to express human somatostatin receptor subtype 2 with an adenoviral vector. Cancer, 94:1298-305.

Rosen LS. 2005. VEGF-targeted therapy: therapeutic potential and recent advances. Oncologist, 10:382-91.

Ruoslathi E and Pierschbacher MD. 1986. Arg-Gly-Asp: a versatile cell recognition signal. Cell, 44:517-18.

Ruoslathi E. 2002. Specialization of tumour vasculature. Nature Rev Cancer, 2:83-90.

Ryan MT, Poston JWS (eds). 2005. A half century of health physics, Lippincott Williams and Wilkins.

Salem R, Thurston KG, Carr BI, et al. 2002. Yttrium-90 microspheres: Radiation therapy for unresectable liver cancer. J Vasc Interv Radiol, 13:223S-9S.

Scott PA, Harris AL. 1994. Current approaches to taargetign cancer using antiangiogenesis therapies. Cancer Treat Rev, 20:393-412.

Sgouros G. 2002. Radiolabeled antibodies, overview. Encyclopedia of Cancer. Elsevier Science.

Sgouros G. 2005. Dosimetry of internal emitters. J Nucl Med, 46:18s-27s.

Sgouros G, Knox SJ, Joiner MC, et al. 2007. MIRD continuing education: Bystander and low dose-rate effects: Are these relevant to radionuclide therapy? J Nucl Med, 48:1683-91.

Sharkey RM, Goldenberg DM. 2005. Perspectives on cancer therapy with radiolabeled monoclonal antibodies. J Nucl Med, 46:115s-27s.

Shukla S, Wu G, Chatterjee M, et al. 2003. Synthesis and biological evaluation of folate receptor-targeted boronated PAMAM dendrimers as potential agents for neutron capture therapy. Bioconjugate Chem, 14:158-67.

Sims DE. 1986. The pericyte: a review. Tissue Cell, 18:153-74.

Snyder AR. 2004. Review of radiation-induced bystander effects. Hum Exp Toxicol, 23:87-9.

Sofou S, Enmon RM, McDevitt MR, et al. 2004a. Multivesicular radioimmunoliposomes with encapsulated Actinium-225 for targeted alphaparticle therapy of intraperitoneal micrometastatic cancer. Cancer Biother Radiopharm, 19:s518-9.

Sofou S, Thomas JL, Lin H-Y, et al. 2004b. Engineered liposomes for potential $\alpha$-particle therapy of metastatic cancer. J Nucl Med, 45:253-60.

Sofou S, Kappel BJ, Jaggi JS, et al. 2007. Enhanced retention of the alphaparticle-emitting daughters of actinium-225 by liposome carriers. Bioconjugate Chem, 18:2061-7.

Sofou S. 2007. Surface-active liposomes for targeted cancer therapy. Nanomed, 2:711-24

Søyland C, Hassfjell SP. 2000. Survival of human lung epithelial cells following in vitro alpha-particle irradiation with absolute determination of the number of alpha-particle traversals of individual cells. Int J Radiat Biol, 76:1315-22.
Stolz B, Weckbeckre G, Smith-Jones PM, et al. 1998. The somatostatin receptor-targeted radiotherapeutic [90y-DOTA-DPhe1, Tyr3] octreotide (90Y-SMT 487) eradicates experimental rat pancreatic CA 20948 tumours. Eur J Nucl Med, 25:668-74.

Supiot O, Gouard S, Charrier J, et al. 2005. Mechanisms of cell sensitization to alpha radioimmunotherapy by doxorubicin or paclitaxel in multiple myeloma cell lines. Clin Cancer Res, 11:7047s-52s.

Supiot S, Thillays F, Rio E, et al. 2007. Gemcitabine radiosensitizes multiple myeloma cells to low let, but not high let, irradiation. Radiother Oncol, 83:97-101.

Thirumamagal BTS, Zhao XB, Bandyopadhyaya AK, et al. 2006. Receptor-targeted liposomal delivery of boron-containing cholesterol mimics for boron neutron capture therapy (BNCT). Bioconjugate Chem, 17:1141-50.

Thorpe PE, Burrows FJ. 1995. Antibody-directed targeting of the vasculature of solid tumors Breast Cancer Res Treat, 36:237-51.

Thurber GM, Zajic SC, Wittrup KD. 2007. Theoretic criteria for antibody penetration into solid tumors and micrometastases. $J$ Nucl Med, 48:995-9.

Tolpin EL, Wellum GR, Dohan FC Jr, et al. 1975. Boron neutron capture therapy of cerebral glyomals: II. utilization of the blood-brain barrier and tumor-specific antigens for the selective concentration of boron in gliomas. Oncology, 32:223-46.

Verschraegen C, Kumagai S, Davidson R, et al. 2003. Phase I clinical and pharmacological study of intraperitoneal cis-bis-neodecanoato( transR, R-1, 2-diaminocyclohexane)-platinum II entrapped in multilamellar liposome vesicles. J Cancer Res Clin Oncol, 129:549-55.

Wachsberger P, Burd R, Dicker AP. 2003. Tumor response to ionizing radiation combined with antiangiogenesis or vascular targeting agents: Exploring mechanisms of Interaction. Clin Cancer Res, 9:1957-71.

Wester HJ, Kessler H. 2005. Molecular targeting with peptides or peptidepolymer conjugates: just a question of size? J Nucl Med, 46:1940-5.

Wilbur DS, Pathare PM, Hamlin DK, et al. 1998. Biotin reagent for antibody pretargeting. 3. synthesis, radioiodination, and evaluation of biotinylated starburst dendrimers. Bioconjugate Chem, 9:813-25.

Wong JYC. 2006. Systemic targeted radionuclide therapy: Potential new areas. Int J Radiat Oncol Biol Phys, 66:S74-S82.

Xu FJ, Pirollo KF, Chang EH. 2001. Tumor-targeted p53-gene therapy enhances the efficacy of conventional chemo/radiotherapy. $J$ Control Release, 74:115-28.

Xu LY, Butler NJ, Makrigiorgos GM, et al. 2002. Bystander effect produced by radiolabeled tumor cells in vivo. Proc Natl Acad Sci USA, 99:13765-70.

Yinghuai Z, Peng AT, Carpenter K, et al. 2005. Substituted carboraneappended water-soluble single-wall carbon nanotubes: new approach to boron neutron capture therapy drug delivery. $\mathrm{J} \mathrm{Am} \mathrm{Chem} \mathrm{Soc,}$ 127:9875-80.

Zaidi H, Sgouros G (eds). 2003. Therapeutic applications of Monte Carlo calculations in nuclear medicine, Bristol, IoP.

Zamenhof RG. 1997. Microdosimetry for boron neutron capture therapy: a review. J Neurooncol, 33:81-92.

Zhang J-S, Liu F, Huang L. 2005. Implications of pharmacokinetic behavior of lipoplex for its inflammatory toxicity. Adv Drug Deliv Rev, 57:689-98.

Znati CA, Rosenstein M, McKee TD, et al. 2003. Irradiation reduces interstitial fluid transport and increases collagen content in tumors. Clin Cancer Res, 9:5508-13. 
